\title{
Personality Assessment Using Multiple Online Social Networks
}

\author{
by
}

\author{
Shally Bhardwaj
}

\author{
Thesis submitted to the \\ Faculty of Graduate and Postdoctoral Studies \\ In partial fulfillment of the requirements \\ For the M.A.Sc. degree in \\ Electrical and Computer Engineering
}

School of Electrical Engineering and Computer Science

Faculty of Engineering

University of Ottawa

(c) Shally Bhardwaj, Ottawa, Canada, 2014 


\begin{abstract}
Personality plays an important role in various aspects of our daily life. It is being used in many application scenarios such as i) personalized marketing and advertisement of commercial products, ii) designing personalized ambient environments, iii) personalized avatars in virtual world, and iv) by psychologists to treat various mental and personality disorders. Traditional methods of personality assessment require a long questionnaire to be completed, which is time consuming. On the other hand, several works have been published that seek to acquire various personality traits by analyzing Internet usage statistics. Researchers have used Facebook, Twitter, YouTube, and various other websites to collect usage statistics. However, we are still far from a successful outcome. This thesis uses a range of divergent features of Facebook and LinkedIn social networks, both separately and collectively, in order to achieve better results. In this work, the big five personality trait model is used to analyze the five traits: openness to experience, conscientiousness, extroversion, agreeableness, and neuroticism. The experimental results show that the accuracy of personality detection improves with the use of complementary features of multiple social networks (Facebook and LinkedIn, in our case) for openness, conscientiousness, agreeableness, and neuroticism. However, for extroversion we found that the use of only LinkedIn features provides better results than the use of only Facebook features or both Facebook and LinkedIn features.
\end{abstract}




\section{Acknowledgements}

Although only my name appears on the cover of this dissertation, many people have contributed to its production. I owe my gratitude to all those people who have made this dissertation possible and because of whom my graduate experience has been one that I will cherish forever.

First and foremost, I would like to express my sincere gratitude to my supervisor, Prof. Abdulmotaleb El Saddik, for the continuous support of my master's study, his patience, motivation, enthusiasm, and immense knowledge. His guidance helped me to overcome many crisis situations during this dissertation. I could not have imagined having a better advisor and mentor.

A very special thanks to my co-supervisor, Dr. Pradeep K. Atrey, who not only supported me during this research but guided me to take many decisions of life. I am fortunate enough that he was always there when I needed him. I appreciate his extensive knowledge and skills in many areas and his collaboration in reviewing my thesis.

I would also like to show my gratitude to Dr. Mukesh Saini who was a constant help throughout my thesis. I am also thankful to him for encouraging the use of correct grammar and consistent notation in my writings and for carefully reading and commenting on countless revisions of this manuscript.

I must thank Dr. Carlisle Adams and Miodrag Boilc who provided me an opportunity to take their graduate courses and shared their vast knowledge with me. A warm thanks to Natacha Chiasson, admission officer at University of Ottawa, for her assistance in my admission procedure. I would also like to thank the School of Electrical Engineering and Computer Science at University of Ottawa, especially Sarah Duncan-Leclair, administrative officer, who kindly dealt with my administrative, technical and financial queries. I am also grateful to the ethics committee including Riana Marcotte and Mlanie Rioux for their tremendous guidance and support throughout the ethics application procedure.

I have been blessed with such a good and friendly group of colleagues in the Discover

and MCR lab. Haolin Guo, Abeer, Basim, Yu Guo, Vahid and Majdi were always there to 
listen to me on many difficult days and played an important supporting role during these past two years. I humbly thank Venu Reddy who provided me with great tips on Matlab tools and many useful software.

Above all, I am grateful to my entire family for their love, care, support, patience and most importantly, having trust in me. My parents offered their encouragement through phone calls every week despite my own limited devotion to correspondence. My brother (Omansh Bhardwaj) and sister (Parul Bhardwaj), who always worked as a strength for me despite of miles of difference and time bounding between us. It would be an understatement to say that, as a family, we have experienced some ups and downs in the past two years. Every time I was ready to quit, they did not let me and I am forever grateful.

I also take this opportunity to thank god for his love, blessing, and for always being my strength and guidance in my difficult time. I might not know where life will take me, but walking with you, God, through this journey has given me tremendous strength. The time when I thought it was not possible for me to survive, you have come up with your magical hands and solved my problem. At last, I can say that I always be patient and smile not because I am stronger, but my Lord you are stronger than all the problems. This dissertation stands as a testament to your unconditional love and encouragement. 


\section{Table of Contents}

\begin{tabular}{ll}
\hline List of Tables & viii
\end{tabular}

\begin{tabular}{ll}
\hline List of Figures & ix
\end{tabular}

Nomenclature $\quad$ xi

List of Symbols $\quad$ xi

\begin{tabular}{ll}
\hline List of Abbreviations & 1
\end{tabular}

\begin{tabular}{llr}
\hline 1 & Introduction & 2
\end{tabular}

1.1 Motivation for Personality Assessment . . . . . . . . . . . . . . . . . . 2

1.2 Thesis Goal and Contribution . . . . . . . . . . . . . . . . . . . . . . 4

1.3 Thesis Organization . . . . . . . . . . . . . . . . . . . 6

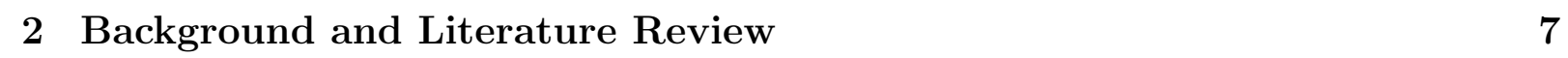

2.1 Personality Theories $\ldots \ldots \ldots \ldots$

2.1 .1 Gordon Allport's trait theory . . . . . . . . . . . . . 8

$2.1 .2 \quad$ Eysenck's three dimensions of personality . . . . . . . . . . . . . . . 9

2.1 .3 The five-factor theory of personality . . . . . . . . . . . . . . . . 9

2.1 .4 The personality theory used in this thesis . . . . . . . . . . . . 11 
2.2 Two OSNs: Facebook and LinkedIn . . . . . . . . . . . . . . . . . . . 11

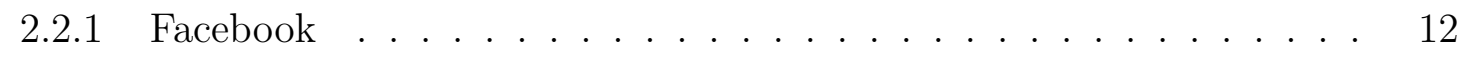

$2.2 .2 \quad$ LinkedIn . . . . . . . . . . . . . . . . . . . . . . . . . . . . . . 13

2.3 Previous Works $\ldots \ldots \ldots \ldots \ldots$

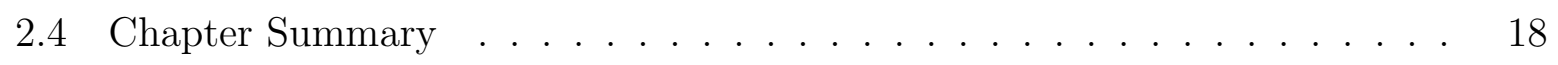

\begin{tabular}{lll}
\hline 3 & Proposed Work & 20
\end{tabular}

3.1 Problem Formulation . . . . . . . . . . . . . . . . . . . . . 20

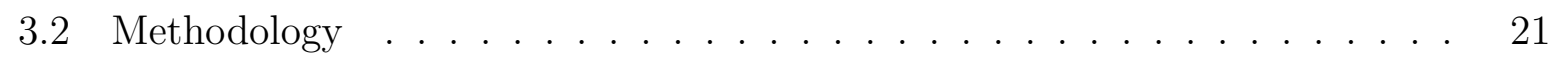

3.2 .1 Information extraction $\ldots \ldots \ldots \ldots \ldots \ldots$

3.2 .2 Feature normalization $\ldots \ldots \ldots \ldots \ldots \ldots$

$3.2 .3 \quad$ Personality modeling $\ldots \ldots \ldots \ldots \ldots \ldots \ldots$

3.3 Experiments and Results $\ldots \ldots \ldots \ldots \ldots$

3.3 .1 Data collection . . . . . . . . . . . . . . . . . 27

$3.3 .2 \quad$ Relationship analysis $\ldots \ldots \ldots \ldots \ldots \ldots$

3.3 .3 Personality assessment results $\ldots \ldots \ldots \ldots$

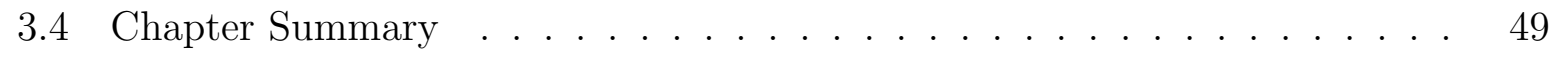

4 Conclusions and Future Research Direction 50

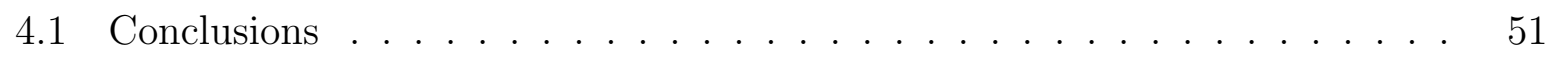

4.2 Future Research Direction $\ldots \ldots \ldots \ldots$

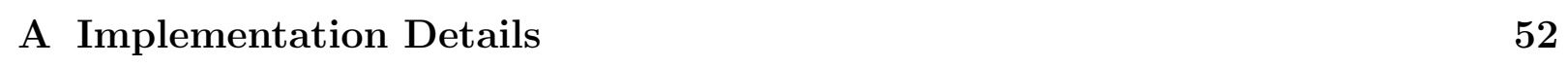

A.1 Data Extraction . . . . . . . . . . . . . . . . . . . . . . . . 52

A.2 Data Analysis . . . . . . . . . . . . . . . . . . . . . 55 
\begin{tabular}{ll}
\hline B User Study & 56
\end{tabular}

\begin{tabular}{lr}
\hline References & 59
\end{tabular} 


\section{List of Tables}

$2.1 \quad$ A comparison of the proposed work with previous works $\ldots .$. . . . . . 18

$3.1 \quad$ Features extracted from Facebook and LinkedIn . . . . . . . . . . . . . . . 23

3.2 Consolidated presentation of the correlation values between OSN features and the big five traits . . . . . . . . . . . . . . . . . . . . 41

3.3 Error $E_{r}$ between $\hat{T}$ and $T$ using Facebook and LinkedIn, individually and collectively, for the big five personality trait trait . . . . . . . . . . . . . . 48

3.4 Personality traits and relation found with features . . . . . . . . . . . . . . 48 


\section{List of Figures}

3.1 The three steps of proposed method: (1) extracting information from OSN (2) feature normalization (3) personality modeling. . . . . . . . . . . . . . 24

3.2 An example of the big five personality test results in percentiles . . . . . . 33

3.3 Correlation coefficient values between Facebook features $\left(f_{11}\right.$ to $\left.f_{19}\right)$ and LinkedIn features $\left(f_{21}\right.$ to $\left.f_{25}\right)$ and the openness trait . . . . . . . . . . . 35

3.4 Correlation values between Facebook features $\left(f_{11}\right.$ to $\left.f_{19}\right)$ and LinkedIn fea-

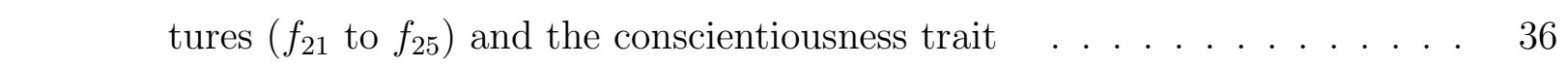

3.5 Correlation values between features $\left(f_{11}\right.$ to $\left.f_{19}\right)$ and LinkedIn features $\left(f_{21}\right.$ to $f_{25}$ ) and the extroversion trait $\ldots \ldots \ldots$. . . . . . . . 37

3.6 Correlation values between features $\left(f_{11}\right.$ to $\left.f_{19}\right)$ and LinkedIn features $\left(f_{21}\right.$ to $f_{25}$ ) and the agreeableness trait . . . . . . . . . . . 38

3.7 Correlation values between features $\left(f_{11}\right.$ to $\left.f_{19}\right)$ and LinkedIn features $\left(f_{21}\right.$ to $f_{25}$ ) and the neuroticism trait . . . . . . . . . . . 38

3.8 Openness Error $E_{r}$ using (i) both Facebook and LinkedIn features (Proposed method) (ii) only Facebook features and (iii) only LinkedIn features . . . . 42

3.9 Conscientiousness Error $E_{r}$ using (i) both Facebook and LinkedIn features (ii) only Facebook features and (iii) only LinkedIn features . . . . . . . . . 44

3.10 Agreeableness Error $E_{r}$ using (i) both Facebook and LinkedIn features (ii) only Facebook features and (iii) only LinkedIn features . . . . . . . . . . . 45 
3.11 Neuroticism Error $E_{r}$ using (i) both Facebook and LinkedIn features (ii) only Facebook features and (iii) only LinkedIn features . . . . . . . . . . . 46

3.12 Extroversion Error $E_{r}$ using (i) both Facebook and LinkedIn features (ii) only Facebook features and (iii) only LinkedIn features . . . . . . . . . . . 47

A.1 Steps involves in data extraction . . . . . . . . . . . . . . . . . 54 


\section{List of Symbols}

A Agreeableness extracted from OSN

$\hat{A} \quad$ Agreeableness extracted from user study

C Conscientiousness extracted from OSN

$\hat{C} \quad$ Conscientiousness extracted from user study

$\mathrm{Cr}$ Correlation matrix

E Extroversion extracted from OSN

$\hat{E} \quad$ Extroversion extracted from user study

$E_{r} \quad$ Error function of personality traits

$F \quad$ The set of features extracted from all OSNs

$F_{s}^{i} \quad$ The set of features extracted from the $i^{\text {th }}$ OSN

$N \quad$ Neuroticism extracted from OSN

$\hat{N} \quad$ Neuroticism extracted from user study

O Openness to experience extracted from OSN

$\hat{O} \quad$ Openness to experience extracted from user study

$T$ The set of big five personality traits extracted from OSN

$\hat{T} \quad$ The set of big five personality traits

obtained with the help of a user study 
$f_{11} \quad$ Number of status updates

$f_{12} \quad$ Number of posts from friends

$f_{13} \quad$ Number of friends

$f_{14} \quad$ Number of pages liked

$f_{15} \quad$ Number of photos

$f_{16} \quad$ Number of tags

$f_{17} \quad$ Number of events

$f_{18} \quad$ Number of groups

$f_{19} \quad$ Number of games

$f_{21} \quad$ Length of text

$f_{22}$ Number of connections

$f_{23} \quad$ Number of skills

$f_{24} \quad$ Number of positive words

$f_{25} \quad$ Number of negative words 


\section{List of Abbreviations}

GUID Globally Unique Identifier

LIWC Linguistic inquiry and word count

MRC Medical research council

OAuth Open authorization

OSN Online social network 


\section{Chapter 1}

\section{Introduction}

This research work intends to explore various online social networks (OSNs) for the purpose of personality assessment. According to the Nielsen company (2010), an individual's total time spent on OSNs in the United States has increased from $15.8 \%$ to $22.7 \%$, while time spent on email has decreased from $11.5 \%$ to $8.5 \%$ [25]. Two-thirds of the online population surf social networking and blogging sites, which accounts of approximately $10 \%$ of the total time spent on the Internet [25]. This pattern is consistent across the world; Facebook has become the 9th most popular brand online. It consists of around 20.5 million users and the average time spent per person on Facebook is 3 hours 10 minutes in a day. In addition to Neilson's study, according to a report by PewResearch on social media update in 2013 [23], $73 \%$ of adults who use the Internet spent a great amount of time on OSNs. Therefore, we can conclude that OSNs are a globally common medium of communication. People share their views, activities, interests, and other daily life attributes on OSNs, which can be quite useful in personality analysis.

\subsection{Motivation for Personality Assessment}

Researchers have recently been obtaining personality information from various sources to perform more accurate personality analysis. They have attempted to relate personality to diverse human characteristics. We discuss the need for personality assessment in different 
scenarios as follows:

Advertisement It is essentially the process of informing the public of producers' activities and products, and increasing market need and company profit. Birknerova et al. [11] suggested that users' buying preferences depend on psychological factors. Each individual's preferences differ based on their unique personality. The role of psychology in advertising is to give their target audience the impression that they absolutely require a given product to fulfill a social or personal need. Thus, it follows that the buying tendencies of a user can be manipulated when the advertiser has his/her personality information [34], [7], and [32].

Ambient environment It is an environment that adjusts itself according to the feel, setting, mood and character of users in it. Such environments provide comfort and convenience to the users. However, each user's comfort and convenience needs differ. For example, some individuals prefer to be in brightly lightened room, whereas others may opt to stay in a dimly lightened and comparatively dark room. Furthermore, people have different preferred music, food and other basic amenities. Therefore, there is a need for personalized ambient environments adjusted according to users' personalities to provide them with a better experience.

Psychology In order to treat various psychological and personality disorders, it is important for psychiatrists to know a patient's personality [21]. This information can be very useful in analyzing a patient's social behavior. It is also helpful in predicting the reaction of a patient at different stages of treatment.

Virtual world Avatars have become very common in various applications such as online gaming, advertisement and education. They are found to be very useful in getting users' attention and giving them an entertaining and high-quality experience. Avatars also play an enormous role in character representation and tend to give a realistic perception to the user [52], [35]. However, current avatars are homogeneous and do not accurately represent a character's personality. Conversely, many personalized avatars behave similarly to the character they represent, thereby enhancing the user 
experience.

Others Personality information can also be useful in determining other factors such as [26]:

- To know important outcomes of life such as divorce, occupational attainment and morbidity. Roberts et al. [48] suggested that personality traits can influence life events such as divorce and occupational attainment. Therefore, it is important to know crucial personality traits (traits that can affect such factors) to avoid the consequences of unexpectedly occurring events in life.

- Hirsh et al. [33] from the University of Toronto presented a study showing that the political preferences of people can be predicted based on their personality. They suggested that it is possible to know political orientation (Conservative, Liberal or National Democratic Party) of a person by analyzing his/her personality [16].

The above discussion justifies the need for developing automated methods to assess the personality of individuals.

\subsection{Thesis Goal and Contribution}

An individual's personality can be defined as a combination of his/her emotional, attitudinal and behavioral responses. Personality assessment has been a widely researched topic in various fields including psychology, medical science and social media. Researchers have also attempted to relate personality traits to diverse human characteristics such as buying tendencies [11], political orientations, study interests, language and career preferences. Furthermore, personality traits can even be associated with human relations and divorce.

Previous studies on personality assessment suggest that there are five main personality traits: openness to experience (for simplicity, we will call it "openness" in the rest of this thesis), conscientiousness, extroversion, agreeableness, and neuroticism. These traits are known as the "big five", a standard that is widely used to measure personality. The 
traditional method of assessing these traits, often employed by psychology researchers, involves a time-consuming process requiring a lengthy questionnaire to be completed by the users. In such cases, the results can be different each time as they are usually dependent on many contextual factors such as mental state of the user and his/her environment.

Recently, a dramatic rise in the use of OSNs such as Facebook, LinkedIn and Twitter has been observed. These OSNs own the behavioral data of millions of users. This has resulted in numerous personality related researches within the social media community, focusing mainly on the relationship between the big five personality traits and OSN features. For example, Bachrach et al. [8] investigated the relationship between user openness and the number of likes and status updates on Facebook.

Although there has been some success in social media driven personality assessment research, the accuracy of the existing approaches is often limited. Furthermore, most of the present works utilize data from a single OSN. Since users often possess multiple OSN accounts, features extracted from multiple OSNs can provide complementary information, which may not be possible to obtain via a single OSN. This can improve the overall accuracy of personality assessment.

The goal of this thesis is to improve the accuracy of automated personality assessment methods using multiple OSNs. To achieve this goal, the thesis presents a new method for assessing an individual's personality. The core idea behind the proposed method is to extract complementary features from more than one OSN account that an individual possesses. The features that are found to have a stronger correlation with the personality traits are then fused to determine the personality traits more accurately compared the earlier approaches where OSNs are used individually. The utility of the proposed method is examined using two popular OSNs, Facebook and LinkedIn. The proposed method is designed to be used to assess the big five personality traits: openness, conscientiousness, extroversion, agreeableness, and neuroticism.

The main contribution of the thesis is twofold:

1. Design and development of an algorithm to access the big five personality traits has been performed using both Facebook and LinkedIn features. 
2. Furthermore, Identifying the crucial features that contributes to big five personality trait identification from multiple OSNs.

\subsection{Thesis Organization}

The rest of this thesis is organized as follows:

- Chapter 2 contains the background details of various personality assessment theories. The chapter also introduces the two OSNs, Facebook and LinkedIn, that are used in this thesis for personality assessment. Also, in this chapter, existing personality assessment works done by the social media community are discussed, and the novelty of the proposed work is highlighted.

- Chapter 3 first presents the formulation of the problem addressed in this thesis. Then, the proposed methodology for personality assessment using multiple OSNs is described in detail. Finally, the chapter presents the experiments, results and analysis in greater detail including data collection, analysis of the relationship between OSN features and personality traits, and personality assessment outcomes.

- Chapter 4 concludes this thesis and proposes the future research directions. 


\section{Chapter 2}

\section{Background and Literature Review}

In this chapter, the background details of various personality theories are provided in Section 2.1. Then, the two OSNs (Facebook and LinkedIn) used in this work are introduced in Section 2.2. Next, in Section 2.3, existing personality assessment works done by the social media community are described and the novelty of the proposed work is discussed. Finally, Section 2.4 presents the chapter summary.

\subsection{Personality Theories}

Personality has a significant influence on the way we perceive outside world, think, feel and relate to other people. The word "personality" itself comes from the Latin word Persona which stands for the theatrical mask to project different roles of a performer [40]. Hall and Linsey claimed in 1957 that there are many definitions of personality as it is articulated by different theorists differently. However, we generally define personality as the combination of characteristics or traits such as emotions, pattern of thoughts, feelings and behaviors unique to an individual [17]. Traits are partly inherent and remain consistent throughout

life, especially after adulthood. However, they can be marginally manipulated with the help of psychiatrists for an individual's amelioration.

In the psychology field, there are different personality theories that have attempted to develop a classification system for individuals who differ in their psychological characteris- 
tics. These personality theories mainly focus on the differences between individuals. Our aim is to define the concept of personality traits in a descriptive manner and to identify the major patterns of behavior by which people can be distinguished or compared.

In the following three subsections, we describe the three different personality theories [18], namely Gordon Allport's Trait Theory, Eysenck's Three Dimensions of Personality, and the Five-Factor Theory of Personality.

\subsubsection{Gordon Allport's trait theory}

In 1936, the psychologist Gordon Allport noticed that an English dictionary consists of more than 4000 words. These words linked to different personality traits and can be useful to analyze a person's behavior. He categorized these personality traits into the following three categories:

- Cardinal traits: These traits can dominate the entire life of an individual to the extent that the person is known specifically for these personality traits. People with such personality traits often become synonymous of such characteristics and traits. However, cardinal traits are rare and develop late in life.

- Central traits: A person's general characteristics that make up the foundation of their personality can be described using the Central traits. These traits are not considered as dominant as Cardinal traits. For example, honest, dishonest, shy, anxious, etc. can be used to describe a person. Central traits are usually inborn characteristics which are difficult to change later in life.

- Secondary traits: We can relate secondary traits to the attitude and preferences of a person. They appear only in a certain situation or under a specific condition. For example: getting anxious when standing in line or getting nervous while speaking to groups. These traits can be improved with practice and psychiatric help. 


\subsubsection{Eysenck's three dimensions of personality}

Another example of a personality trait theory is Eysenck's three dimensions of personality. This theory was proposed by British psychologist Hans Eysenck and it has three universal traits [24]. He believed that many traits are hereditary or inborn and can be shaped by evolutionary forces. These three traits are sufficient to define a person's characteristics and are given below [12]:

- Introversion/Extroversion: Introversion involves directing attention toward the inner experiences of a person. Introverts tend to turn inward and focus more on internal thoughts, moods and feelings rather than seeking external stimulation from their environment. They prefer spending time alone, usually avoid social gatherings and are generally quiet and reserved. Conversely, extroversion involves attention to the outside world. Extroverts are considered sociable, outgoing, tend to have more friends, and like to spend their time with groups of people rather than staying alone. On a scale, introversion is on the low end extroversion is on the high end.

- Neuroticism/Emotional stability: Eysenck measured this trait on the scale of moodiness and even-temperedness. Neuroticism refers to an individual's tendency to be emotionally unstable and get upset easily. Low neuroticism indicates stable and calm behavior.

- Psychoticism: After studying people suffering from various mental illnesses, Eysenck added one more personality dimension to his trait theory which is called as Psychoticism. Psychoticism refers to an individual's tendency to find difficulty dealing with reality. Such individuals are antisocial, manipulative, hostile and unsympathetic to others.

\subsubsection{The five-factor theory of personality}

The five-factor theory of personality, also known as the big five theory, is the most important and widely accepted theory [1]. This theory consists of a hierarchical categorization 
of five personality dimensions:

- Openness: (inventive/curious vs. consistent/cautious). Openness reflects a tendency or degree of intellectual curiosity, art, creativity and the novelty and variety a person has. It also defines the extent of imagination and independence that a person comprises. Here, imagination does not mean that a person is not realistic but that he/she is open to consider new ways of doing things. They know how to balance between existing and new approaches. However, there are some disagreements between theorists about how to interpret openness, which is sometimes referred to as "intellect". People with a high degree of openness tend to be imaginative and liberal in their opinions and thoughts. Poets and artists may be considered as typical examples of high openness scorers [17].

- Conscientiousness: (efficient/organized vs. easy-going/carefree) Conscientiousness reflects a person's tendency to act dutiful, responsible, organized and self disciplined. They aim for achievement and generally have very high standards for work. Conscientious people prefer to be organized and well prepared rather than being spontaneous. They have a strong sense of direction, and self-control at work and in their personal life as well[20].

- Extroversion: (outgoing/energetic vs. solitary/reserved) Extroverts and introverts represent opposite ends of a key personality dimension. Extroverts are outgoing and sociable whereas introverts prefer to spend their time alone and are generally considered reserved. However, this does not conclude that introverts are unfriendly and suffer from social anxiety; they just do not seek external opportunities for excitement. Extroverts are more active, energized and optimistic. Although they prefer group settings and want to be surrounded by people, but they also maintain their privacy [12].

- Agreeableness: (friendly/compassionate vs. analytical/detached) Agreeableness can be defined as the ability to be compassionate and cooperative rather than being suspicious and antagonistic to others. It also measures a person's trusting and helpful 
nature. People displaying this personality dimension are friendly to almost everyone and are always ready to help. They possess the primary facets of trust, modesty, forwardness, and tender-mindedness. Agreeable people are best known for their warm and tolerant behavior [17].

- Neuroticism: (sensitive/nervous vs. secure/confident). Neuroticism is the emotional and mental stability of a person under adverse situations. People who score high on neuroticism can be very sensitive to a stimulus and become nervous easily in a stressful situation. They also suffer more from anxiety. Conversely, individuals who score lower on neuroticism are considered consistently calm and stable. Instability of emotions can sometimes result in feelings of guilt, anger, mental stress and anxiety. It is believed that people with high neurotic scores tend to have more heart attacks and psychological disorders than those who have a low neurotic score [51]. This indicates that neurotic individuals tends to have high range of negative emotions [42], [41].

\subsubsection{The personality theory used in this thesis}

In this thesis, we use the 'big five' theory of personality assessment. The big five traits of an individual can be obtained using a "big five personality test" developed by the psychologist Oliver D. John [37], at the University of California, Berkeley. This test consists of nearly 50 questions and takes 10-15 minutes to measure personality traits on a Likert scale. It provides the traits score in the range of 0 to 100, where 0 represents a low value and 100 represents a high value of a trait.

\subsection{Two OSNs: Facebook and LinkedIn}

Facebook and LinkedIn are the online social medium for people to interact with others. Initially, on the Internet we had social tools such as chat forums and newsgroups. Today it has evolved to include many additional components, such as blogs, fantasy environments, and OSNs. 
Recently, a rigorous escalation has been seen in the use of OSNs. OSNs are making an increasingly important influence on our daily lives. OSNs have become a popular medium of communication and they allow users to share their daily life information and receive updates from friends and their favorite celebrities. The OSN profile comprises crucial information which can be used to identify the behavioral pattern of a user. In 2000, Hamburger published a study suggesting that an individual's pattern of Internet usage and their personality are related. He suggested that every person has a different pattern of usage of these networks which is influenced by the personality of that person. Furthermore, in 2010, Back et al. 9] proposed that Facebook profiles reflect the actual personality of users, not self idealized ones.

We categorize these OSNs in two different groups: (i) Formal OSNs, and (ii) Informal OSNs. The OSNs that are used for general communication purposes, entertainment activities, sharing views, and connecting with friends are considered as Informal OSNs, e.g. Facebook. On the other hand, networks that are used for professional, business and educational purposes are refereed to as Formal OSNs, e.g. LinkedIn. Due to the fact that

both groups of OSNs provide a range of diverse and important information, it is advisable to utilize both for a better personality assessment outcome.

In this thesis, we use one formal (LinkedIn) and one informal OSN (Facebook). An overview of these two OSNs is given as below.

\subsubsection{Facebook}

Facebook is considered to be the world's largest OSN with more than one billion users. It was first founded in 2004 by Mark Zuckerberg [44] and then introduced to the world in 2006. It is used not only as a medium of communication between people with different geographical locations, but also for entertainment, marketing, advertisement and various other activities. The profile owners can easily share their views, photos and videos with their friends. One of the advantages of Facebook is that it allows an unlimited number of uploads for posts, photos and videos unlike other OSNs such as Photobucket and Flicker. Furthermore, the privacy settings of Facebook offer a great deal of flexibility to users who 
want to share content with a particular person, a group or their entire friend list. It also provides a variety of other features such as messaging and audio-video calling.

\subsubsection{LinkedIn}

LinkedIn is a business-oriented OSN which is used by employers, employees, and students for professional networking. It was founded in 2002 and launched in 2003. It currently has 259 million users from more than 200 countries. Any person who has an email address can become a member of LinkedIn and share his/her expertise and accomplishments. This OSN is accessible in 20 different languages, with English being the most common. As of 2013, it is reported to have nearly 65.5 million Unites States visitors and 184 million visitors globally. Unlike Facebook, LinkedIn connections refer to the contacts who have same level of relationship. Users can upload their resume and seek jobs and business opportunities. For identification purposes, users can post their own photos and also see other's photos. It brings potential employers and job seekers to the same platform to facilitate business networking. They can share their achievements, awards, success and records, and can also congratulate others for the same. Consistent with these developments, employers are increasingly using LinkedIn for pre-selection and recruitment purposes.

LinkedIn allows people to send or receive online recommendations for their professional abilities and characteristics. It introduces people to potential colleagues and employers in their own field as well as in other fields. LinkedIn also helps users find job postings on its website from various employers. The greatest benefit is that the users can find job postings exclusive to LinkedIn that are not advertised elsewhere because they require more than one or two recommendations through LinkedIn. Furthermore, a person may get the opportunity to meet people who are already working in the area or company where he/she wants to work. This helps to know more about the area and prepare for interview [22]. 


\subsection{Previous Works}

In this section, we review existing personality assessment research works done by the social media community. We found that there are many psychology works that relate personality traits to online social behavior. They found some evidences that personality and online behavior do have link to each other. For example: people scoring on high neuroticism often use more social media services like Facebook, Twitter and blogs [19], [30]. However, some studies found a negative correlation between neuroticism and overall Internet usage [54]. Moreover, people with emotional instability seems to have additive behavior on the Internet [31, 443, 45]. A study done by Blumer et al. describes briefly about relation of all five traits and online behavior [13]. This implies that there is a inconsistency in previous findings. There are also numerous studies exhibit on obtaining these traits from OSNs automatically using machine learning algorithms. However, the accuracy of personality trait detection from OSNs is typically low. We found various works that used features from only formal or informal OSNs to obtain the personality traits. However, the use of features from both dimensions of OSNs has not been explored yet, which we do in this thesis. Below, we discuss existing works that are related to the work proposed in this thesis.

In 2000, Amichai-Hamburger and Ben-Artzi [4] presented a study that says that Internet use is related to personality. They analyzed the Internet use patterns of different men and women with Extrovert and Neuroticism traits. According to this study, men were found to be positively linked to leisure services for extroversion, whereas neuroticism was negatively correlated to information services. However, for women, the results were the opposite for these services. These outcomes are important because they indicate that personality is an essential and highly relevant aspect of Internet behavior analysis. Similarly, Amichai-Hamburger et al. [2] successfully showed the relation between Internet usage and personality. They suggested that personality is indicated by which is an important factor in understanding why people behave the way they do on the Internet. For a long time,

personality was ignored by the designers who decided the future of the Internet. One reason for this may be that designers have mainly focused on technological advancement. 
Amichai-Hamburger et al. argued that the only way to improve the lack of personality perspective on the Internet is to have a cooperative effort from both Internet designers and psychologists. Since the Internet is powered by human interaction, we cannot understand the Internet without knowing the personality of the user [3]. In addition to the above studies, in 2008, Amichai-Hamburger et al. [5] studied the link between personality and nostalgic websites. In this work, the authors concluded that extroverts made more use of social services on nostalgic websites than introverts. Furthermore, the authors suggested that people's surfing of nostalgic websites affects their pattern of behavior in society. This indicates that users behave in society the same way as they do in online social interactions. This suggests that extroverts also maintain their social dominance on OSNs as compared to introverts.

In addition to the above studies, Ross et al. [49] found that conscientiousness is negatively related to the number of Facebook activities, as conscientious people generally limit the use of Facebook in order to meet deadlines and obligations. The results of this work were re-examined by Amichai-Hamburger et al. [6]. They also suggested that individuals with conscientious personalities generally have higher numbers of friends. The authors used the analysis of covariance (ANCOVA) in order to examine the effect of conscientiousness on Facebook use. It was found that the individuals who scored higher on the conscientiousness trait were found to demonstrate less use of the picture upload feature than individuals who scored lower.

This hypothesis was supported by the results. Individuals who scored higher on the trait of conscientiousness were found to have a higher number of friends (Mean $=147.8$ Standard Deviation $(\mathrm{SD})=112.6$ ) than individuals who scored lower on the trait of conscientiousness (Mean $=112.72 \mathrm{SD}=82.49)$. In this study, all the participants were asked to complete NEO-PI-R, a self report measure, to assess five personality traits. NEO-PI-R is a widely used self report measure of the big-five personality traits. It consists of 240 questions which are scored on a Likert scale from strongly agree to strongly disagree. Furthermore, users' Facebook information was evaluated and analyzed. Specifically, the following information was analyzed: basic information and personal/demographic information such as gender, 
birth dates, and hometown, activities, interests, hobbies; and education and work related information such as university and position description. The main difference between [49] and [6] was in their methodological approach.

In addition to these works, the big five model was used by Mitja et al. [9] as a reference. They showed that the Facebook profile reflects the actual personality of an individual rather than self-idealization. The relation between OSN features and the big five traits was discussed earlier by Golbeck et al. [28] as well. They examined the correlation between the big five traits and the linguistic features of Twitter such as frequency of swear words, perceptual words and demographic information from the user's profile. They also used nonlinguistic features such as number of followers, number of followings, number of replies, and density of social network.

To analyze the linguistic features of a user's profile they used linguistic inquiry and word count (LIWC), a linguistic program and a Medical research council (MRC) database consisting of a psycholinguistic database of 150,000 words with the linguistic and psycholinguistic features of each word. In addition, two regression models: Gaussian process and ZeroR were used to train and test the system with the Waikato Environment for Knowledge Analysis (Weka) software. Their results indicate that the frequency of swear words has a negative correlation with perceptual words and a positive correlation with words surrounding social processes. However, the correlation found between personality traits and Twitter features was very small, i.e. only $11 \%$ to $18 \%$ of actual personality. A similar work using the Facebook features was done by Golbeck et al. [29]. A recent study by Quercia et al. [47] found that extroversion is positively related to the number of friends on Facebook as well as in the real world. In a similar study, they also found that extroversion is a positive predictor of Twitter usage.

Another study by Kartelj et al. [39] was based on corpus data which included both text and meta data of Twitter. The mechanism used here exhibited two sets of features: LIWC and the MRC database, which showed the correlation to personality characteristics. The LIWC database software package evaluated the word and correlated it to personality characteristics, whereas the MRC database found the frequency of use of the words. Simi- 
larly, in another work, Wald et al. [56] incorporated both demographic and text, where 31 demographic and 80 text-based independent attributes from Facebook were used. Three algorithms, linear regression, REPTree and decision tables were used to compare results. The REPTree algorithm showed $74 \%$ accuracy for openness, $50 \%$ accuracy for agreeableness and $34.5 \%$ average for overall personality traits and proved to be better than the other two algorithms.

In addition to the studies from Kartelj et al. [39] and Wald et al. [56], Bachrach et al. [8] also used machine learning algorithms to predict personality from Facebook. They used features such as friends, groups, likes, tags, status and photos. The data of nearly 180000 users was collected using the mypersonality application which allows users to complete a five-factor model questionnaire. Their study was conducted in two steps: 1) find the features correlated to personality, and 2) apply a machine learning algorithm to the data of correlated features. They suggested that openness is correlated to the number of likes whereas conscientiousness, which reflects a person's tendency to be disciplined and organized, is negatively related to the number of likes, but positively related to the number of photos uploaded. However, their conscientiousness results contradict the results found by Hamburger and Vinitzky [6] in 2010. Furthermore, agreeableness was not found to be related to Facebook features, which again contradicts the results of Hamburger and Vinitzky [6], who found that agreeableness is negatively related to the number of photos uploaded.

In another work, Verschuren [55] employed five psychology students to provide their rating on 65 users' LinkedIn profiles. These ratings were later validated with the help of a user study. Contrary to their manual approach, we provide an automated method to determine personality traits using LinkedIn features.

Note that the above mentioned works and several other similar works (using Twitter and other blogs) used only a single OSN in their studies. Compared to past works on OSNbased personality assessment, the novelty of our work lies in the following two aspects: we,

- employ multiple OSNs, and

- use LinkedIn features for personality assessment. 
Table 2.1: A comparison of the proposed work with previous works

\begin{tabular}{|c|c|c|c|c|}
\hline The work & $\begin{array}{l}\text { Personality } \\
\text { traits exam- } \\
\text { ined }\end{array}$ & OSNs used & Approach used & Remarks \\
\hline $\begin{array}{l}\text { Amichai- } \\
\text { Hamburger } 4\end{array}$ & $\begin{array}{l}\text { Extroversion } \\
\text { and Neuroti- } \\
\text { cism }\end{array}$ & $\begin{array}{l}\text { Internet leisure, so- } \\
\text { cial and, informa- } \\
\text { tion services }\end{array}$ & Manual survey & $\begin{array}{l}\text { Demonstrated a link between } \\
\text { personality and the Internet }\end{array}$ \\
\hline $\begin{array}{l}\text { Amichai- } \\
\text { Hamburger et } \\
\text { al. [5] }\end{array}$ & Extroversion & $\begin{array}{l}\text { HEVREA (nostal- } \\
\text { gic website) }\end{array}$ & Manual & $\begin{array}{l}\text { Found no effect of extroversion } \\
\text { on the use of nostalgic websites }\end{array}$ \\
\hline Ross et al. 49 & Big five traits & Facebook & Manual survey & $\begin{array}{l}\text { Extroverts were found to have } \\
\text { more groups and neurotic peo- } \\
\text { ple were found to have more } \\
\text { Facebook wall posts, less pho- } \\
\text { tos and tags }\end{array}$ \\
\hline $\begin{array}{l}\text { Amichai- } \\
\text { Hamburger and } \\
\text { Vinitzky [6] }\end{array}$ & Big five traits & Facebook & Automated & $\begin{array}{l}\text { Did not find a strong correla- } \\
\text { tion between people's person- } \\
\text { ality and their Facebook activ- } \\
\text { ities }\end{array}$ \\
\hline Golbeck et al. 28 & Big five traits & Twitter & $\begin{array}{l}\text { Automated (Pear- } \\
\text { son correlation } \\
\text { analysis and re- } \\
\text { gression analysis) }\end{array}$ & $\begin{array}{l}\text { Used linguistic features of } \\
\text { Twitter (found a weak correla- } \\
\text { tion) }\end{array}$ \\
\hline Golbeck et al. 29 & Big five traits & Facebook & $\begin{array}{l}\text { Automated (Pear- } \\
\text { son correlation } \\
\text { analysis) }\end{array}$ & $\begin{array}{l}\text { Found weak correlation be- } \\
\text { tween people's personality and } \\
\text { their Facebook activities }\end{array}$ \\
\hline Verschuren 55 & Big five traits & LinkedIn & $\begin{array}{l}\text { Manual (with the } \\
\text { help of psycholo- } \\
\text { gists' ratings) }\end{array}$ & $\begin{array}{l}\text { No LinkedIn features were used } \\
\text { and no methodological ap- } \\
\text { proach was adopted) }\end{array}$ \\
\hline Proposed work & Big five traits & $\begin{array}{l}\text { Facebook and } \\
\text { LinkedIn }\end{array}$ & Automated & $\begin{array}{l}\text { Facebook and LinkedIn } \\
\text { features were fused }\end{array}$ \\
\hline
\end{tabular}

A comparison of the past works and the proposed work is provided in Table 2.1.

\subsection{Chapter Summary}

In this chapter, three personality theories, namely Gordon Allport's trait theory, Eysenck's three dimensions of personality, and the five-factor theory of personality were described. Also, a brief description of the two OSNs (Facebook and LinkedIn) that are used in this work is provided. Furthermore, the previous works on personality assessment are discussed 
from different perspectives and the novelty of the work proposed in this thesis is highlighted. 


\section{Chapter 3}

\section{Proposed Work}

In this chapter, a detailed description of the method proposed in this thesis is presented. First, in Section 3.1, we formally state the research problem addressed in this thesis. Next, in Section 3.2, the proposed methodology is described in detail. Experiments and results are presented in Section 3.3 . Finally, Section 3.4 presents the chapter summary.

\subsection{Problem Formulation}

We formulate the problem of combined multiple OSNs based personality assessment as follows: Let $G$ be a function that receives inputs from a set of $n$ OSNs, $S$, where $S=$ $\left\{S_{1}, S_{2}, \ldots, S_{n}\right\}$.

Several features are extracted from these OSNs such as the number of friends, number of posts, and number of likes, which relate to the personality of an OSN user. In this research, we use two popular OSNs: Facebook and LinkedIn.

Let $F$ be the set of features extracted from all OSNs, which can be defined as the union of a set of features extracted from an individual OSN, i.e.,

$$
F=\cup_{i=1}^{n} F_{s}^{i}
$$

where $F_{s}^{i}$ is the set of features extracted from the $i^{\text {th }}$ OSN. 
Personality traits that a person may have are represented by $T$, where $\mathrm{T}$ can be either of the following personality traits; $O, C, E, A$, or $N$ which represent openness, conscientiousness, extroversion, agreeableness and neuroticism, respectively. These five personality traits are dependent on the features $F$ that are extracted from different OSNs, $S$, and each trait is denoted by a single value, which is computed using a function $G$. The function $G$ takes $F$ as input and provides personality traits $T$, i.e.,

$$
T=G(F)
$$

Note that the value of $T$ is computed by combining all the features $F$ using the function $G$, details of which are provided in Section 3.2 .3 .

Our goal of this work is to find $G$ such that the following error function $E_{r}$ is minimized:

$$
E_{r}=|\hat{T}-T|
$$

where $\hat{T}$ is the personality traits obtained with the help of a user study, which is also

considered as ground truth. Here, $\hat{T}$ can be either $\hat{O}, \hat{C}, \hat{E}, \hat{A}$, or $\hat{N}$. The value of $\hat{T}$ is calculated by user study. A lower value of the error function indicates improved accuracy.

\subsection{Methodology}

The proposed personality assessment method is shown in Figure 3.1. The method includes three basic steps: information extraction, feature normalization, and personality modeling. The first step is to extract different features as numeric and text information from multiple OSNs such as Facebook $\left(F_{s}^{1}=\left\{f_{11}, f_{12} \ldots f_{19}\right\}\right)$ and LinkedIn $\left(F_{s}^{2}=\left\{f_{21}, f_{22} \ldots f_{25}\right\}\right)$. The next step is to normalize these feature values, followed by the calculation of personality by fusing normalized features. Based on feature information, we map features and personality traits to find crucial features (feature which helps to determine and reduce the personality traits) to determine the personality. Furthermore, we combine all crucial features that depends on personality for each personality trait correspondingly. This fusion of social network features helps to minimize the error. The details of each step are given in the following subsections. 


\subsubsection{Information extraction}

OSNs have multi-modal information in the form of numbers (number of likes), images, videos and texts (status, comments). In our case, we focus on mainly numeric and text based information as it can be obtained easily and accurately. In 1989, Larsen \& Katelaar suggested that positive and negative words are associated with extroversion and neuroticism respectively [41]. Therefore in our research we also considered these two linguistic features (positive and negative words) to predict personality traits. While the usage of statistics and basic information can be directly used numerically, we need to apply a psychological linguistic software on text in order to find linguistic characteristics such as length of text, a negative or positive words, which are used to analyze the attitude of a person. In our case, we use an LIWC text analysis program that helps to count and sort words into psychological meaningful categories [53]. It also estimates the count of positive and negative words used in text. However, text is only collected from LinkedIn but not Facebook. The reason behind using LinkedIn text is that it reflects the experience level, academic achievements, and awards. These factors describe the range of discipline of a person. In addition to that, we extract a set of features from the $\operatorname{OSNs}_{(}\left(F_{s}^{i}\right.$, for $i=1$ to $\left.n\right)$. The extracted features for Facebook and LinkedIn are shown in Table 3.1. For the sake of simplicity, we assume that the feature values in the set $F$ have already been converted to numeric values. 
Table 3.1: Features extracted from Facebook and LinkedIn

\begin{tabular}{|l|l|}
\hline Feature number & Facebook features \\
\hline$f_{11}$ & Number of status updates \\
$f_{12}$ & Number of posts from friends \\
$f_{13}$ & Number of friends \\
$f_{14}$ & Number of pages liked \\
$f_{15}$ & Number of photos \\
$f_{16}$ & Number of tags \\
$f_{17}$ & Number of events \\
$f_{18}$ & Number of groups \\
$f_{19}$ & Number of games \\
\hline Feature number & LinkedIn features \\
\hline$f_{21}$ & Length of text \\
$f_{22}$ & Number of connections \\
$f_{23}$ & Number of skills \\
$f_{24}$ & Number of positive words \\
$f_{25}$ & Number of negative words \\
\hline
\end{tabular}




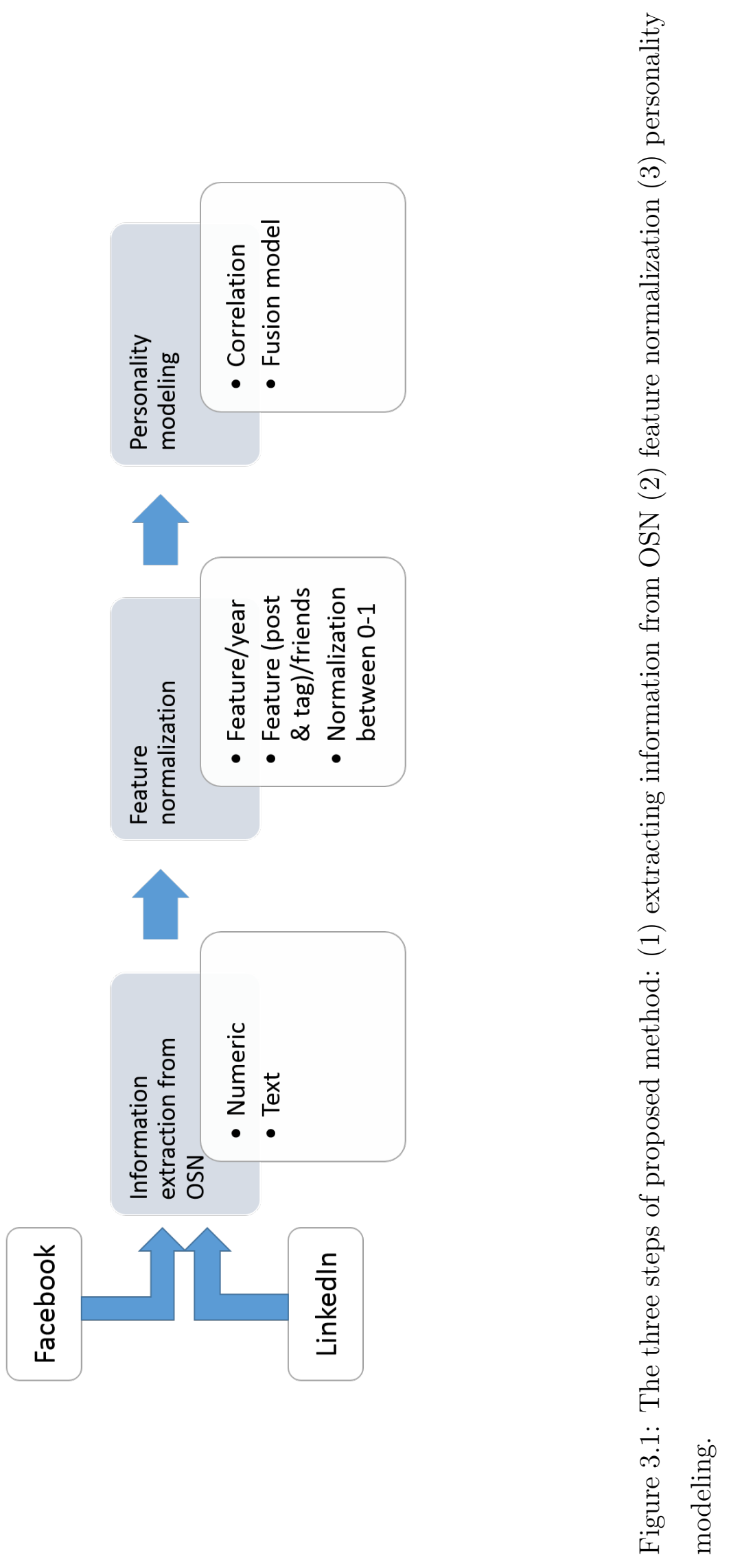




\subsubsection{Feature normalization}

The normalization of features is an important step in our method due to the fact that each feature has its own range varying from zero to an unknown value, which is generally a large number. To fuse different features together, we need to bring them on common measuring scale and normalize them on a feature values between 0 and 1 . We formally describe the normalization steps below:

1. Divide features by the number of years in order to obtain the feature value per year. For example, if a user has $f$ status updates so far, divide it by the number of years the user has been active on the OSN to obtain $f^{\prime}=f /$ years.

2. For user specific features, such as posts and tags, further divide the features by the number of friends to obtain $f^{\prime \prime}=f^{\prime} /$ friends.

3. Normalize each feature by scaling between 0 and 1 . Here, we consider the average of features to be 0.5 . Below average and above average values map to [0-0.5] and [0.5-1], respectively. We obtain the normalized feature value $\hat{f}$ of a given feature value $f^{\prime \prime}$ using the following equation:

$$
\hat{f}= \begin{cases}\frac{1}{2}+\frac{\left(f^{\prime \prime}-\operatorname{avg}\left(f^{\prime \prime}\right)\right)}{2 \times\left(\max \left(f^{\prime \prime}\right)-\operatorname{avg}\left(f^{\prime \prime}\right)\right)}, & f^{\prime \prime}>\operatorname{avg}\left(f^{\prime \prime}\right) \\ \frac{f^{\prime \prime}}{2 \times \operatorname{avg}\left(f^{\prime \prime}\right)}, & f^{\prime \prime}<\operatorname{avg}\left(f^{\prime \prime}\right) \\ \frac{1}{2}, & \text { otherwise }\end{cases}
$$

In the above equation, avg and $\max$ denote the functions that return the average and maximum values of $f^{\prime \prime}$, respectively.

This normalized feature matrix is used to determine personality traits (as described in the next section).

\subsubsection{Personality modeling}

A feature can be related to a given personality trait in different ways. To study the nature of the relationship, we need to find the correlation between the personalities of the OSN users 
and the corresponding feature values. Let us assume that $\mathrm{Cr}=\left\{c r_{i j} \mid 1 \leq i \leq m, 1 \leq j \leq l\right\}$ is a correlation matrix between $m$ personality traits and $l=|F|$ features.

The features that affect the personality will have significant values in the correlation matrix. In the experiments we found that the correlation value of each feature is different for each trait. From the experiments we learned that any feature with an absolute correlation value from $\pm(0-1)$ can affect the corresponding personality traits. Therefore, we found correlated crucial features with the trial and error method. The set of correlated features obtained is denoted as $F_{c r} \in F$. We have removed the personality trait subscript for clarity. A personality trait can be related to the feature positively or negatively depending on the sign of the correlation coefficient.

Let us denote positively correlated features as $F_{c r}^{+}$and negatively correlated features as $F_{c r}^{-}$, i.e., $F_{c r}=F_{c r}^{+} \cup F_{c r}^{-}$. To combine these positively and negatively correlated features, we use the following formula:

$$
T^{\prime}=\sum_{\forall f_{i} \in F_{c r}^{+}} \bar{\omega}\left(f_{i}\right) \hat{f}_{i}+\sum_{\forall f_{j} \in F_{c r}^{-}} \bar{\omega}\left(f_{j}\right)\left(1-\hat{f}_{j}\right)
$$

where $T^{\prime}$ is the normalized personality trait value between 0 and 1 , and $\bar{\omega}\left(f_{k}\right)$ is a function that returns the weight of feature $f$ which is obtained by scaling the absolute value of the corresponding correlation coefficient such that the sum of all weights for features in $F_{c r}$ is unity. The standard personality trait value $T$ is obtained by multiplying $T^{\prime}$ by 100 , i.e., $T=100 T^{\prime}$.

For example: we first extract two OSN features i.e. number of likes (from Facebook) and number of skills (from LinkedIn), which are measured 20 and 30. In order to normalize the number of likes feature, we divide it by the number of years, say 2, resulting into a value 10. Further, this resulted value of number of likes, 10, is then normalized on a scale 0-1 using Equation 3.4 considering the maximum value of number of likes, say 100. In our implementation, we used the maximum value obtained within 31 users. Therefore, we divide 10 by 100 and finally get the normalized value of the number of likes as 0.1. Similarly, the normalized value of the number of skills feature will be 30/100 (i.e. 0.3). 
For LinkedIn features, we do not use the first and the second step of normalization due to the reason that LinkedIn does not disclose information about the joining year. After normalizing all the features, we find the correlation between the personality trait values and the normalized values of different features. Let us consider, for openness to experience trait, we obtained a score 30 on a scale 0-100 with the help of user study, and it was found correlated to both the features; number of likes and number of skills. We then combine the values of these features using Equation 3.5, where we first convert all negative values into positive values by subtracting it by 1 and then multiply each normalized features to its weight (correlation values) to further add all feature values, to find the value of openness of experience.

\subsection{Experiments and Results}

The main goal of our experiments is to find the relation between features and personality traits, and to show that the error in personality detection can be reduced using combined features of multiple OSNs. In this section, we will first discuss the data collection for our experiments (in Section 3.3.1). Then we will analyze data to explore the relation between features and the big five personality traits (in Section 3.3.2. Finally, in Section 3.3.3. we will detect these personality traits using (1) only Facebook features, (2) only LinkedIn features, and (3) features from both Facebook and LinkedIn.

\subsubsection{Data collection}

\section{Participants}

The data of 31 people from different locations was collected. Some of them were graduate students and others were employed at different organizations. Participants were both females and males of different ages from 18 to 50. We have involved people from different fields to bring diversity to the data set.

The identities of all the participants were kept anonymous during the personality test 
using Globally Unique Identifier (GUID). GUID is a 128 bit integer number which is used to name or to identify a resource. The generation algorithm of GUID is unique enough that if 1,000,000,000 GUIDs per second were generated for 1 year the probability of a duplicate would be only 0.5 . In our case, we have only 31 users, therefore we can rely on the GUID numbers.

\section{Procedure and measurement}

The collected data was of the following two types: i) OSN based, and ii) user study based. We describe them as follows:

Facebook and LinkedIn data collection The user information uploaded on Facebook and LinkedIn was collected using a PHP SDK and MySQL and was analyzed using Matlab. The detailed description of the implementation is given in Appendix A. There are several features used for research, which are given in Table 3.1. With this, we found patterns of OSN usage of different people.

The rationale for choosing Facebook and LinkedIn features is as follows:

1. Rationale for choosing Facebook features: It is well known that Facebook is currently the most widely used OSN for communication and sharing views. Therefore, we expect that it can provide useful information about a person which can help to find the personality traits. We chose to use the following Facebook features due to the reasons given below:

(a) $f_{11}$ (Number of status updates) : We postulate that extroverted and agreeable individuals should have a large number of status updates. The reason behind that is that extroverts tend to talk more and share their feelings more often than others [12] [18]. Therefore, they should have a positive relation to the status update feature.

(b) $f_{12}$ (Number of friends): We suggest that $f_{12}$ (number of friends) should be correlated to extroversion and agreeableness due to the reason that extroverts like to make new friends and meet people [12]. In addition to that, 
people like agreeable individuals and wants to be their friends due to their warm and helpful nature [38] [1].

(c) $f_{13}$ (Number of posts by friends): We hypothesize that agreeable individuals should have more posts on their time-lines from their friends due the reason that they are the most liked personality among all other [38].

(d) $f_{14}$ (Number of likes): We postulate that the individuals who have high openness scores would be more creative, like to explore new things, and appreciate new inventions. They get bore easily because of their tendency to pursue new interests, they tend to have more likes on Facebook [17.

(e) $f_{15}$ (Number of photos): We believe that extroverted and conscientious personalities would have more photos. The idea behind this relation is that extroverts are outgoing people. Moreover, they love to share their life events with their friends which leads them to upload more photos. On the other hand, conscientious individuals, rather than spending their time on status updates or other online activities, prefer to work due to their hard working and disciplined nature [17]. However, they like to update and share their awards with their friends and other people. This behavior leads them to upload more photos to share their awards and achievements. Our assumption is supported by the result obtained by Bachrach et al. [8] where they showed that more conscientious people tend to upload more photos.

(f) $f_{16}$ (Number of tags): The people having high scores on agreeableness would have more tags from friends because they are the most liked people and usually have more friends [17, 38] and [1].

(g) $f_{17}$ and $f_{18}$ (Number of events and number of groups respectively): We surmise that neurotic individuals would have less $f_{17}$ (number of events) and more OSN groups online [38] [1]. Neurotic individuals tend to have low self esteem and confidence which leads them to avoid social gatherings, parties and events. In addition, in real scenarios neurotic individuals tends to have feelings of insecurity, inferiority, and shyness [17]. Therefore, this is a very presumptuous that in order to feel better and compensate, perhaps 
they involve with more online groups.

(h) $f_{19}$ (Number of games): We propose that $f_{19}$ (number of games) should have a link with openness and neuroticism. The idea behind the relation between $f_{19}$ (number of games) and openness is that open people like to play different types of games because they tend to change their interests very often and move on the new things. On the other hand, neurotic individuals like to spend their time playing games to avoid stress and anxiety [45], [14]. We do not find any reason to link games with other personality traits. Note that although extroverts are fun-loving and athletic. They prefer to play more outdoor games instead of playing online games. Conscientious people like to spend their time in more meaningful ways such as doing their professional work rather than playing games.

2. Rationale for choosing LinkedIn features: We noticed that LinkedIn is one of the most widely used OSNs by professionals and students. Hence, we believe that the information available from LinkedIn can be vital in the analysis of the big five personality traits of a person.

(a) $f_{21}$ (Length of text): From [15], we identify that personality is also related to a person's achievements, success, rewards and experience. In order to extract this information, we use the length of text from the LinkedIn profile as it usually reflects that individuals having more achievements, rewards and experience tend to have lengthy profiles. We believe that the $f_{21}$ (length of text) can be related to various features such as conscientiousness, openness, and extroversion. Note that conscientious people tend to have more achievements and awards. In addition to that we assumed that individuals who are open to experience, have a great vocabulary and like to present their ideas in more expressive ways, write lengthier text. Moreover, extroverts who like to speak more and has many things to say should have more length of text in writing as well.

(b) $f_{22}$ (Number of connections): We postulate that the $f_{22}$ (number of connec- 
tions) reflects the level of extroversion in a person. The reason behind this is that on LinkedIn users have only professional connections, as opposed to having both personal and professional friends on Facebook. It is noticed that on Facebook people can have their own family, relatives and family friends as their friends. In this way it is difficult to determine if a person is actually an extrovert. On the other hand, LinkedIn connections are often legitimate and professional which can show how extroverted a person is in the external world.

(c) $f_{23}$ (Number of skills): We propose that a high $f_{23}$ (number of skills) should be related to openness and conscientiousness. Openness to experience individual have a wide range of interests [14]. On the other hand, conscientious individuals are extremely devoted to their work and success. We believe that those who have more skills will have higher conscientiousness, as conscientious individuals are more organized and achievement-oriented in their lives [10, which leads them to learn more professional skills.

(d) $f_{24}$ (Positive words): It is believed that the words people use in their daily lives can reflect important aspects of their social and psychological worlds. The words they use can reveal a person's behavior, attitude, and overall personality [46]. Therefore, we postulate that positive emotions should be linked to extroversion, agreeableness and neuroticism. We know that extrovert individuals are highly optimistic and exhibit positive emotions [27], [42]. We also assume that agreeableness would have a positive relation to the number of positive words because agreeable individuals are generally calm, cooperative, helpful and sympathetic to others [10]. In contrast, neurotic individuals should have a negative correlation to positive words because they are more prone to negative emotions [14].

(e) $f_{25}$ (Negative words): Neurotic individuals would also use a large $f_{25}$ (number of negative words) because they are more prone to negative feelings such as anger, hostility, guilt and anxiety [20].

The above postulates will be validated with experiments in Section 3.3 .2 . 
User study based data collection A user study was performed with 31 people. All participants were asked to complete the personality assessment test with the widely used big five model self-report measure. The test consists of 50 questions scored on a five-point Likert scale from strongly disagree (1) to strongly agree as (5). The survey questionnaire is provided in Appendix B. Figure 3.2 shows an example of the big five personality test results.

\section{Text to numeric data conversion}

The two types of data that we utilize are: numeric data and text data. In our implementation, we get the numeric data from OSNs. For example: 2 number of likes (from Facebook) or 5 number of skills (from LinkedIn). Because it is difficult to interpret and fuse the textual data, we converted it to numeric values. In order to analyze the textual data collected from Facebook and LinkedIn, we used LIWC linguistic software.

Over the last three decades, researchers have provided evidence to suggest that systematic associations between personality and usage of words differ for each personality. [57]. More recently, Lecobelli et al. used LIWC for a large scale study on personality and linguistic features [36]. The authors suggested that personality is related to $f_{24}$ (positive

words) and $f_{25}$ (negative words). Hence, in our work, we used LIWC to find $f_{21}$ (length of text), $f_{24}$ (positive words) and $f_{25}$ (negative words). 


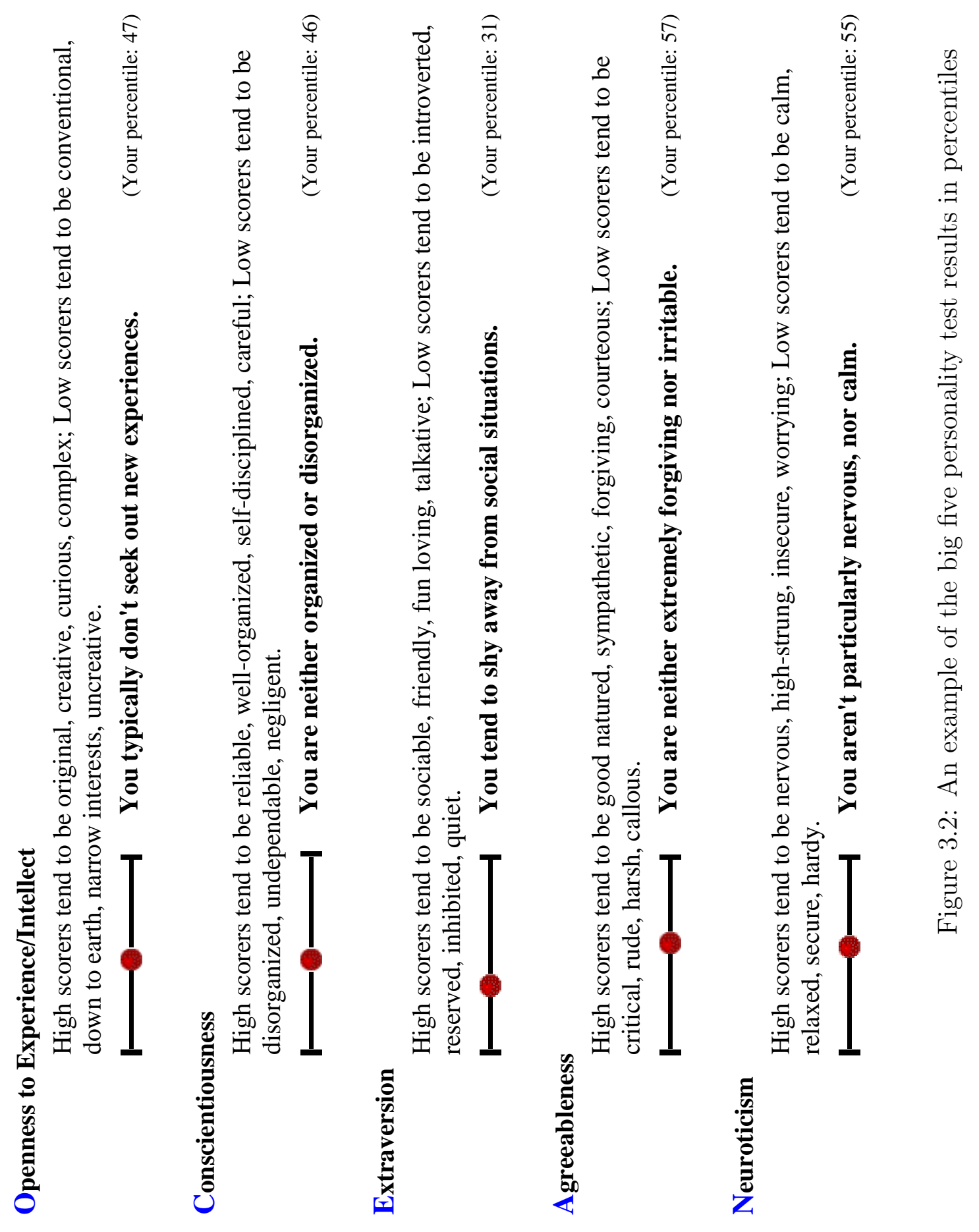




\subsubsection{Relationship analysis}

In order to study the relationship between the collected OSN features and the personality traits, we calculated the Pearson's correlation coefficient between the features and the big five personality traits values using the data of 31 people.

To correlate the personality traits and OSN features, we first extracted the actual personality of the users with the help of the user study which consisted of the big five personality test, as explained earlier in Section 3.3.1. The results of the user study are normalized between 0 and 100. Here, normalization is important because the maximum value of each feature is different. In order to analyze them together there is a need to bring them on the same scale. In addition, each Facebook and LinkedIn feature is normalized to its maximum value. However, the $f_{22}$ (number of connections) obtained from LinkedIn is normalized to 500 because the maximum LinkedIn connection that can be extracted is 500. Also, we can only have a maximum of 60 skills on LinkedIn. Therefore, the skills feature was normalized to 60 .

The relationship between 9 Facebook and 5 LinkedIn features, and the big five personality traits is discussed as follows.

\section{Relationship between Facebook and LinkedIn features and openness trait}

The correlation value between Facebook and LinkedIn features and the openness trait is shown in Figure 3.3. In the given figure, we can see that the most prominent correlation exists between openness and the Facebook and LinkedIn features. It is found that the $f_{14}$ (number of likes) have $C r=0.86$. In other words, people having more $f_{14}$ (number of likes) on Facebook are found to be more open. Another significant correlation value for Facebook features has been seen with $f_{12}$ (number of games) $(\mathrm{Cr}=0.68)$. On the other hand, openness has a positive correlation with two LinkedIn features, $f_{22}$ (length of text) and skills, with correlations of 0.32 and 0.33 respectively. It is also noticed that result shows a negative correlation between openness and $f_{24}$ (number of positive words). However, this 'Cr' result contradicts the behavior of open personalities. In Section 3.3 .3 we 


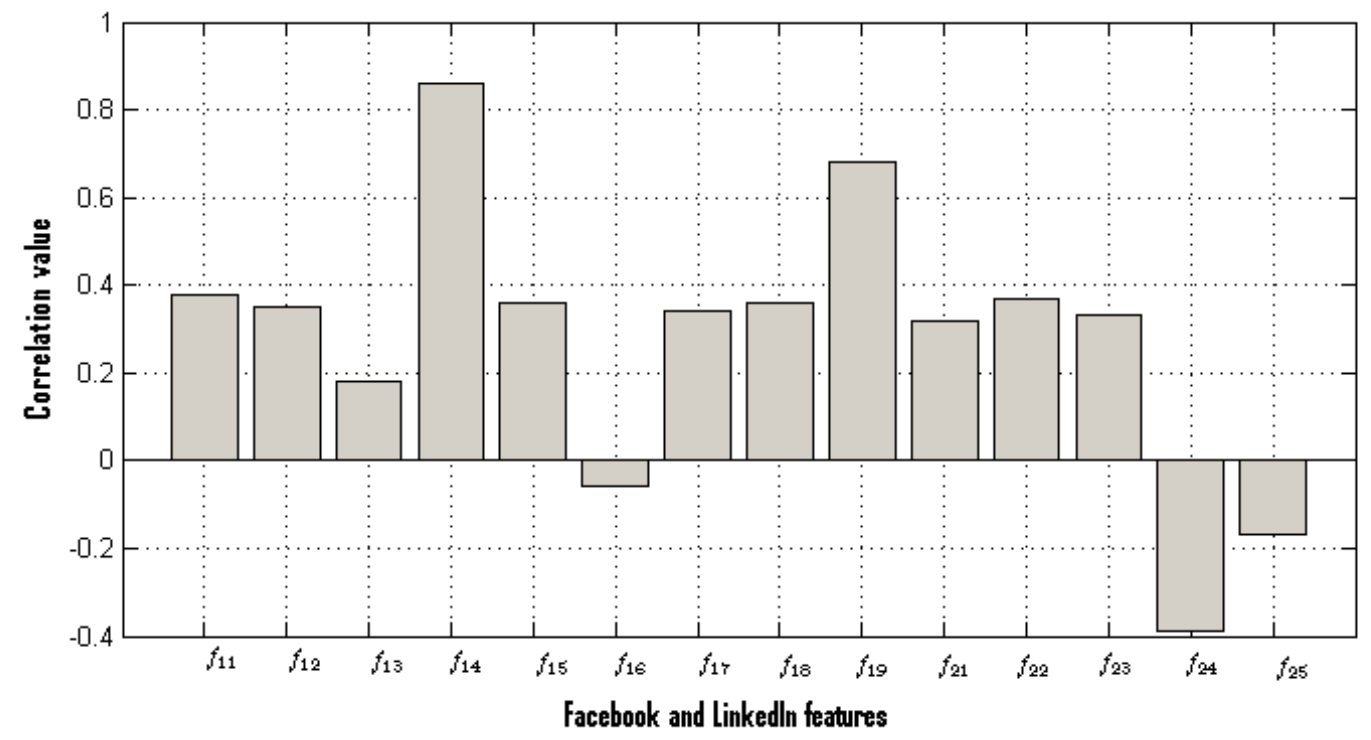

Figure 3.3: Correlation coefficient values between Facebook features $\left(f_{11}\right.$ to $\left.f_{19}\right)$ and LinkedIn features $\left(f_{21}\right.$ to $\left.f_{25}\right)$ and the openness trait

examined the true relation and analyzed that $f_{25}$ (positive words) are not useful to predict openness. Therefore, we do not consider $f_{25}$ (positive words) to analyze openness.

\section{Relationship between Facebook and LinkedIn features and conscientiousness trait}

The relationship between the conscientiousness trait and the Facebook and LinkedIn features is shown in Figure 3.4 in terms of the correlation coefficient. It can be seen in the figure that there are both positive and negative correlation values. The conscientiousness trait has a high positive correlation $(C r=0.83)$ with Facebook feature $f_{15}$ (number of photos). It is also found that conscientiousness shows a relatively high positive correlation with three LinkedIn features: $f_{21}$ (length of text), $f_{22}$ (number of connections), and $f_{23}$ (number of skills) given as $C r=0.75, C r=0.61$, and $C r=0.70$. Adversely, it has a negative correlation $(\mathrm{Cr}=-0.24)$ with the Facebook feature $f_{13}$ (number of friends).

Therefore, it is evident from Figure 3.4 that the personality trait of conscientiousness depends on the above mentioned highly correlated Facebook (mainly the $f_{15}$ (number of photos)) and LinkedIn (mainly $f_{21}$ length of text, $f_{22}$ connections and $f_{23}$ skills) features. 


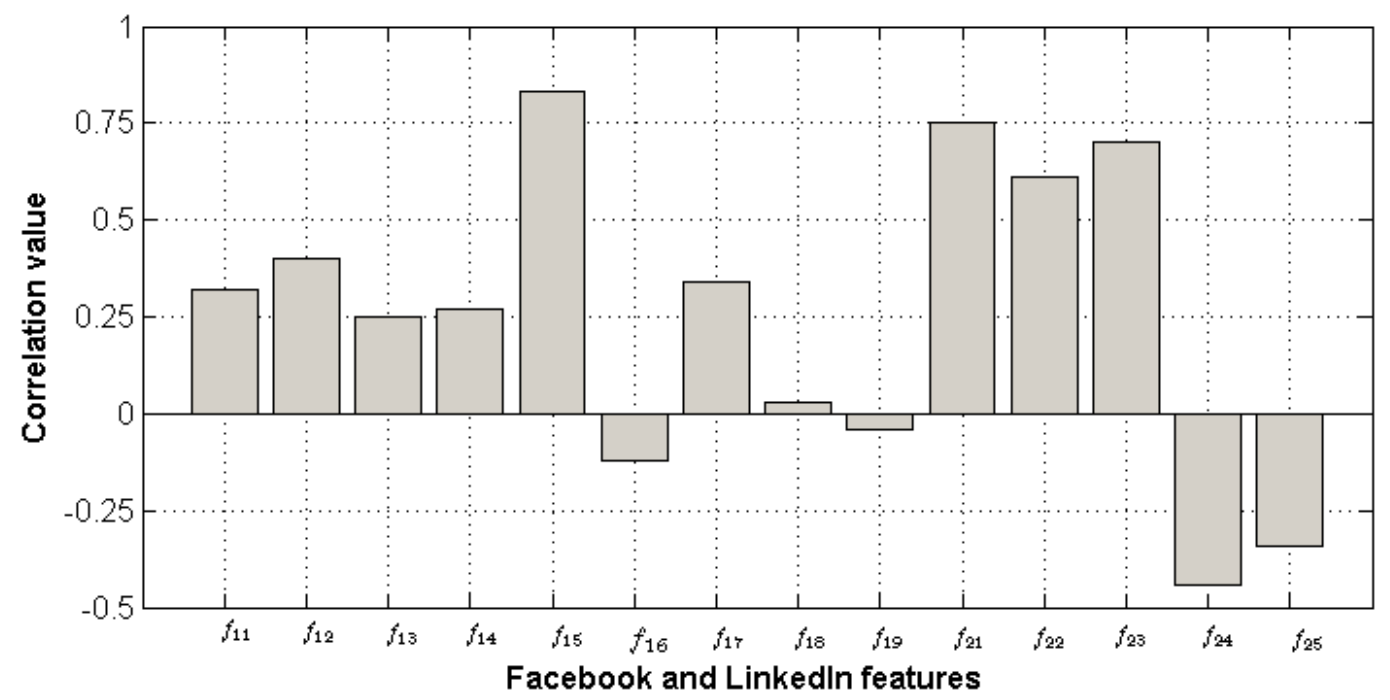

Figure 3.4: Correlation values between Facebook features $\left(f_{11}\right.$ to $\left.f_{19}\right)$ and LinkedIn features $\left(f_{21}\right.$ to $\left.f_{25}\right)$ and the conscientiousness trait

In later experiments, we will show that by incorporating these features, we can significantly reduce the error in assessing the conscientiousness trait.

\section{Relationship between Facebook and LinkedIn features and extroversion trait}

Figure 3.5 shows the correlation between the extroversion trait and the features from Facebook and LinkedIn. Similar to the previous two traits, we can see that there exists both positive and negative correlations. As can be seen in the figure, the Facebook features $f_{12}$ (number of friends) and $f_{15}$ (number of photos) have a relatively high positive correlation ( $C r=0.38$ and $C r=0.48$ respectively), followed by the LinkedIn feature $f_{21}$ (length of text) with $C r=0.78$ and $f_{22}$ (number of connections) with $C r=0.91$. We can see that some of the above results contradict the postulates given in Section 3.3.1. For example: we postulated that extroverts should have a positive correlation to number of status updates, however we do not find a high correlation with it. Therefore we will consider and examine both postulated and highly correlated features in Section 3.3 .3 to find out how these features contribute in reducing the error for assessing the extroversion personality trait. 


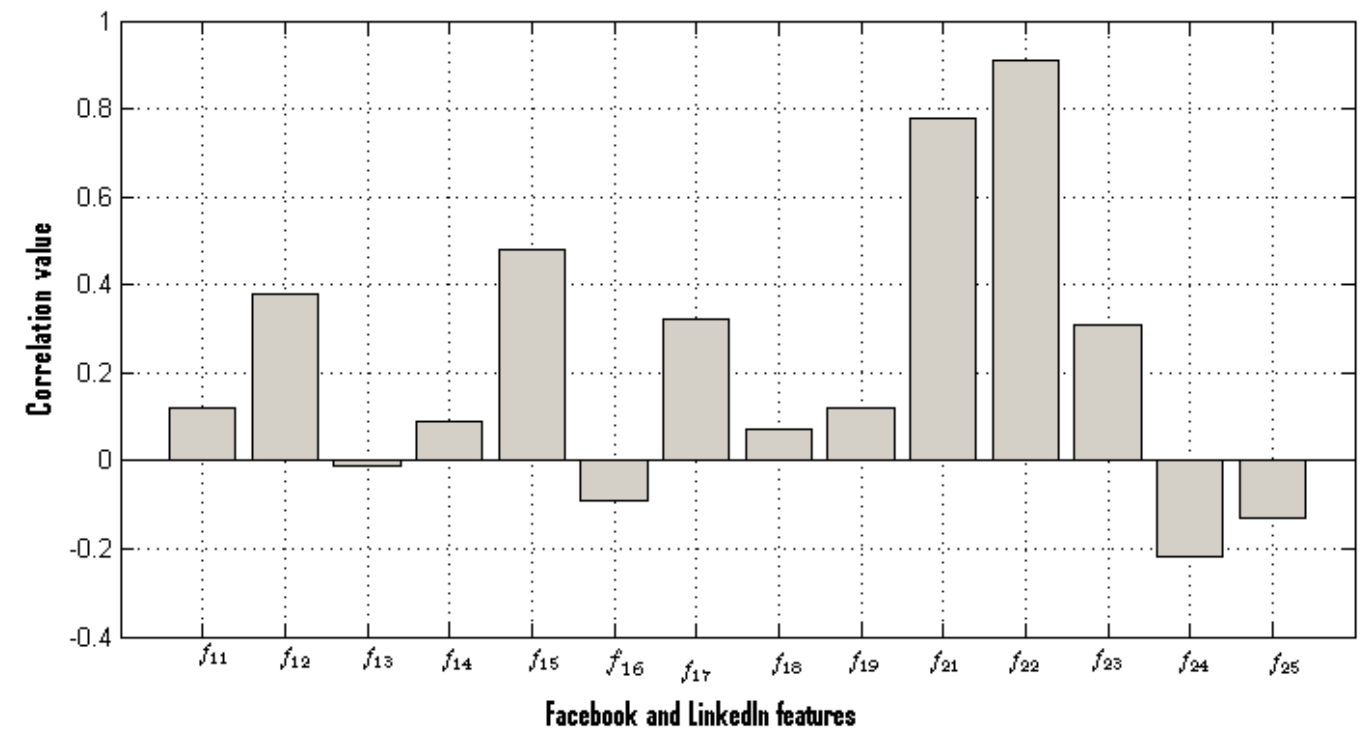

Figure 3.5: Correlation values between features $\left(f_{11}\right.$ to $\left.f_{19}\right)$ and LinkedIn features $\left(f_{21}\right.$ to $\left.f_{25}\right)$ and the extroversion trait

\section{Relationship between Facebook and LinkedIn features and agreeableness per- sonality trait}

As shown in Figure 3.6, the agreeableness trait has both positive and negative correlations with features. We found that there is a reasonably high positive correlation between the agreeableness trait and Facebook features such as $C r=0.61$ with $f_{11}$ (number of status updates), $\mathrm{Cr}=0.59$ with $f_{12}$ (number of friends), and $f_{15}$ (number of photos). While these results support our postulates, we also found a new feature $f_{14}$ (number of photos) with a high correlation. Furthermore, skills from LinkedIn provided a high positive $\mathrm{Cr}=0.75$ and $f_{24}$ (positive words) a relatively high negative $C r=-0.44$ partially supported by postulates. We believed that $f_{12}$ (positive words) should have a positive correlation with agreeableness, however results show this to be untrue. This result contradicts the basic behavior of agreeable individuals. Therefore, we further examine it in Section 3.3.3. 


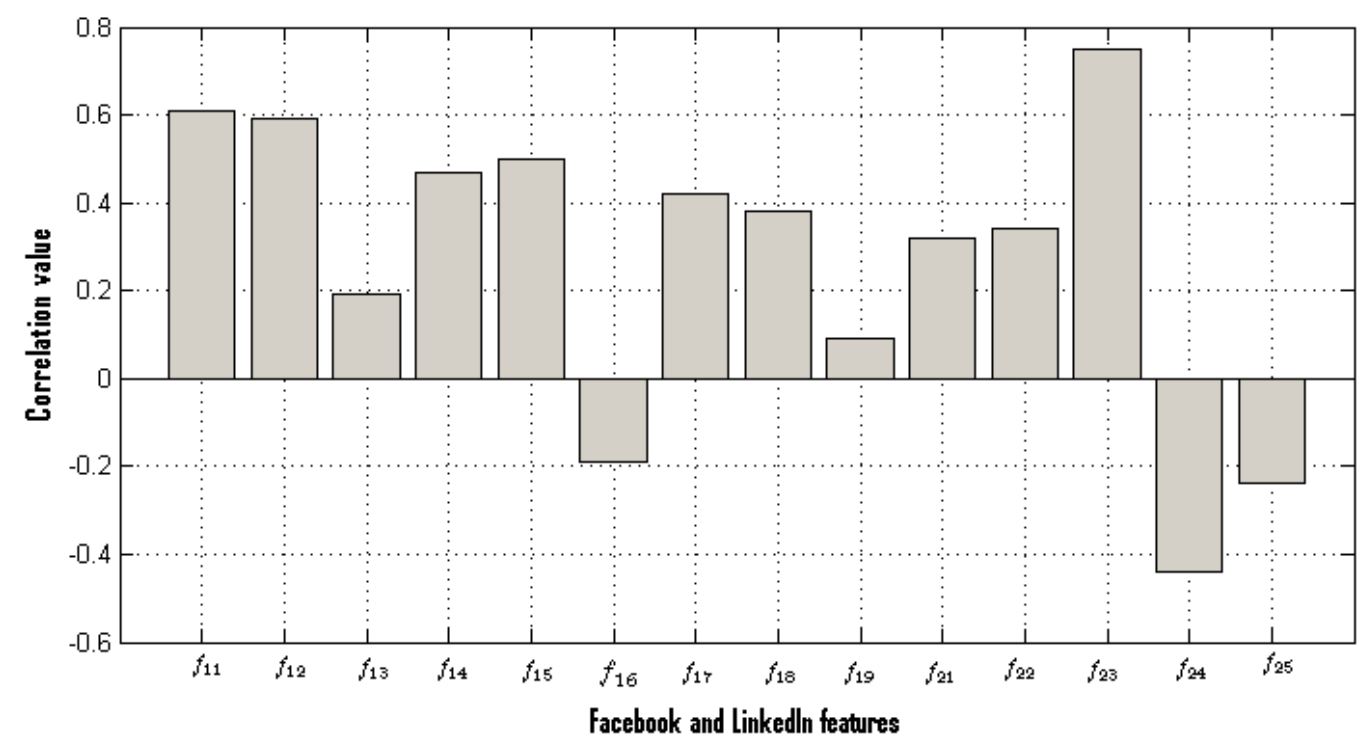

Figure 3.6: Correlation values between features $\left(f_{11}\right.$ to $\left.f_{19}\right)$ and LinkedIn features $\left(f_{21}\right.$ to $\left.f_{25}\right)$ and the agreeableness trait

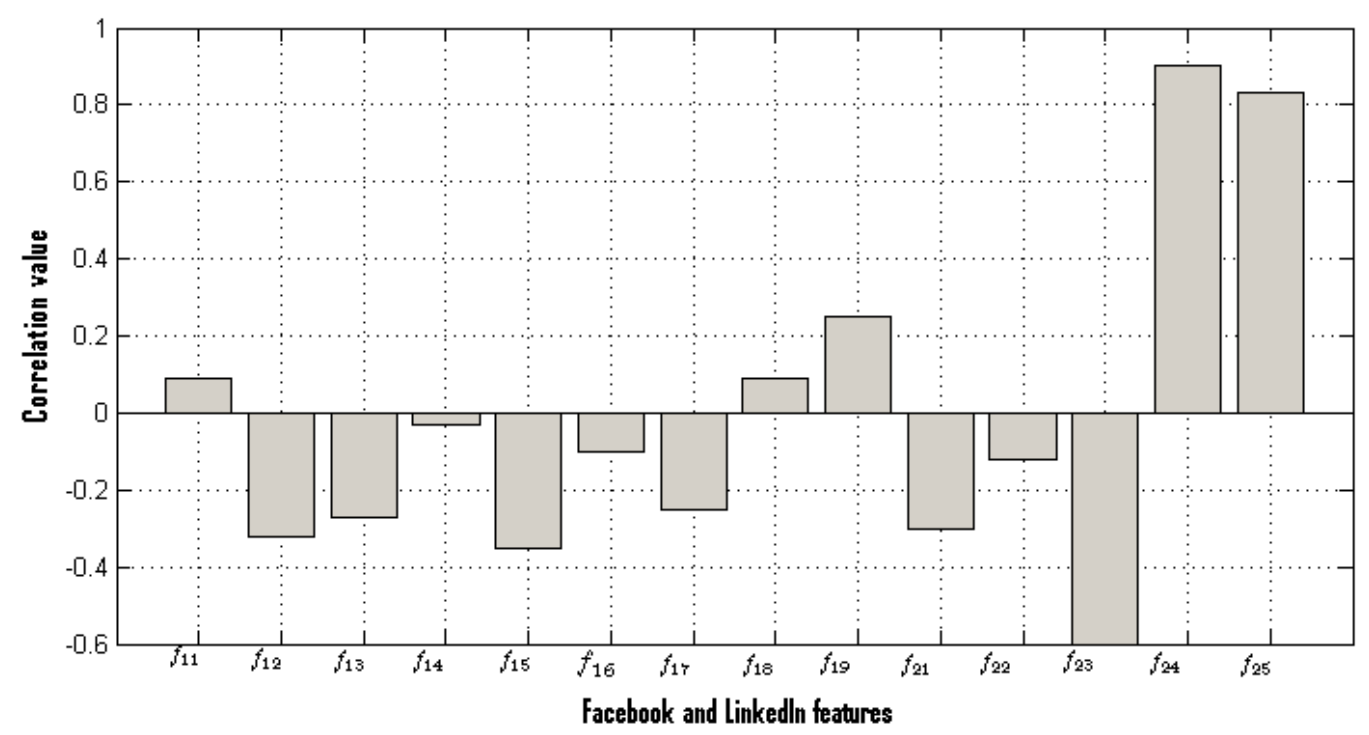

Figure 3.7: Correlation values between features $\left(f_{11}\right.$ to $\left.f_{19}\right)$ and LinkedIn features $\left(f_{21}\right.$ to $\left.f_{25}\right)$ and the neuroticism trait 


\section{Relationship between Facebook and LinkedIn features and neuroticism trait}

Similar to the other four traits, the neuroticism trait is also both positively and negatively correlated with various features from Facebook and LinkedIn, as can be seen in Figure 3.7. We observed that it is positively correlated with Facebook feature $f_{19}$ (number of games) with $C r=0.25$ and negatively correlated to $f_{12}$ (number of friends), $f_{13}$ (number of posts), $f_{15}$ (number of photos), and $f_{17}$ (number of events) with $C r=-0.32, C r=-0.27$, $C r=-0.35$, and $C r=-0.25$ respectively. These results are partially supported by our postulates where the $f_{17}$ (number of events) and $f_{19}$ (number of games) were expected to have good correlation. Interestingly, the neuroticism trait is found to have a stronger correlation with the other three Facebook features ( $f_{12}$ (number of friends), $f_{13}$ (number of post), $f_{15}$ (number of photos)) as well. Therefore, these features are examined in Section 3.3.3. Furthermore, we noted very high positive correlation of neuroticism and LinkedIn features which are: $f_{24}$ (positive words) and $f_{25}$ (negative words) with $\mathrm{Cr}=0.90$ and $\mathrm{Cr}=0.83$. These outcomes partially support our postulates mentioned in the previous section. Surprisingly, neuroticism was found to have a positive correlation with positive words. In the next section, we will further examine the effect of these features on the neuroticism trait.

\subsubsection{Personality assessment results}

\section{Feature extraction and normalization}

To determine the big five personality traits, nine Facebook features i.e. $f_{11}, f_{12}, f_{13}, \ldots, f_{19}$ and five LinkedIn features i.e. $f_{21}, f_{22}, f_{23}, \ldots, f_{25}$ (as shown in Table 3.1) were used. Since the number of years a user has been on Facebook varies for different users, we normalized the Facebook features based on the number of years. Note that there are some features that can further vary based on other features, for example, $f_{12}$ and $f_{16}$ can vary based on the number of friends in the user circle. Therefore, further normalization of Facebook features was performed on the basis of $f_{12}$ number of friends.

In our experiments we used five LinkedIn features (i.e. $f_{21}$ to $f_{25}$ ). Note that these 
features were also normalized to their maximum values. However, unlike Facebook features, LinkedIn features were not normalized based on the number of years. This is because a LinkedIn profile does not provide any information on the year of the user's joining the OSN.

\section{Validation}

In this step, we find the difference between the vectors $\hat{T}$ and $T$, i.e., the value of the trait achieved from the user study and the value achieved from the OSN feature set. In other words, we compute the error $E_{r}$ (as per Equation 3.3). In order to find the accuracy of the proposed method for all five traits, we used median, mean and mode as average functions (in Equation 3.4). While choosing the Facebook and LinkedIn features for determining the value $T$ of the five traits, we considered the correction between the OSN features and the traits. For the sake of simplicity, the consolidated presentation of the correlation values is provided in Table 3.2 ,

Overall, we found that for the openness, conscientiousness, agreeableness and neuroticism traits, the error $E_{r}$ is less when both Facebook and LinkedIn features are used together as compared to the error when Facebook and LinkedIn features are used separately. However, for one trait (extroversion), this is not the case. In other words, for the extroversion trait, LinkedIn features work quite well and adding Facebook features does not offer any benefit. In order to understand the reason behind this we tried to analyze the behavior of both social media features. As we know, for the extroversion assessment we found the following crucial features: $f_{11}$ (number of status updates), $f_{12}$ (friends), $f_{21}$ (length of text), $f_{22}$ (connections) and $f_{25}$ (negative words). Out of these features, some behave in a similar manner such as friends from Facebook and connections from LinkedIn, and some differently such as status updates. $f_{21}$ (length of text) and $f_{24}$ (number of negative words), which can be common in both Facebook and LinkedIn, behave in a similar fashion. However, in our research we only use text from LinkedIn due to the unavailability of Facebook textual data. We believe that a similar feature can provide better results if used collectively from two OSNs. Therefore, we suggest that there is a possibility to further improve the result 
Table 3.2: Consolidated presentation of the correlation values between OSN features and the big five traits

\begin{tabular}{|l|l|l|l|l|l|l|}
\hline OSN & Features & $O$ & $C$ & $E$ & $A$ & $N$ \\
\hline Facebook & $f_{11}$ & 0.38 & 0.32 & 0.12 & 0.61 & 0.09 \\
\hline Facebook & $f_{12}$ & 0.35 & 0.4 & 0.38 & 0.59 & -0.32 \\
\hline Facebook & $f_{13}$ & 0.18 & 0.25 & -0.01 & 0.19 & -0.27 \\
\hline Facebook & $f_{14}$ & 0.86 & 0.27 & 0.09 & 0.47 & -0.03 \\
\hline Facebook & $f_{15}$ & 0.36 & 0.83 & 0.48 & 0.5 & -0.35 \\
\hline Facebook & $f_{16}$ & -0.06 & -0.12 & -0.09 & -0.19 & -0.1 \\
\hline Facebook & $f_{17}$ & 0.34 & 0.34 & 0.32 & 0.42 & -0.25 \\
\hline Facebook & $f_{18}$ & 0.36 & 0.03 & 0.07 & 0.38 & 0.09 \\
\hline Facebook & $f_{19}$ & 0.68 & -0.04 & 0.12 & 0.09 & 0.25 \\
\hline LinkedIn & $f_{21}$ & 0.32 & 0.75 & 0.78 & 0.32 & -0.3 \\
\hline LinkedIn & $f_{22}$ & 0.37 & 0.61 & 0.91 & 0.34 & -0.12 \\
\hline LinkedIn & $f_{23}$ & 0.33 & 0.7 & 0.31 & 0.75 & -0.6 \\
\hline LinkedIn & $f_{24}$ & -0.39 & -0.44 & -0.22 & -0.44 & 0.9 \\
\hline LinkedIn & $f_{25}$ & -0.17 & -0.34 & -0.13 & -0.24 & 0.83 \\
\hline
\end{tabular}




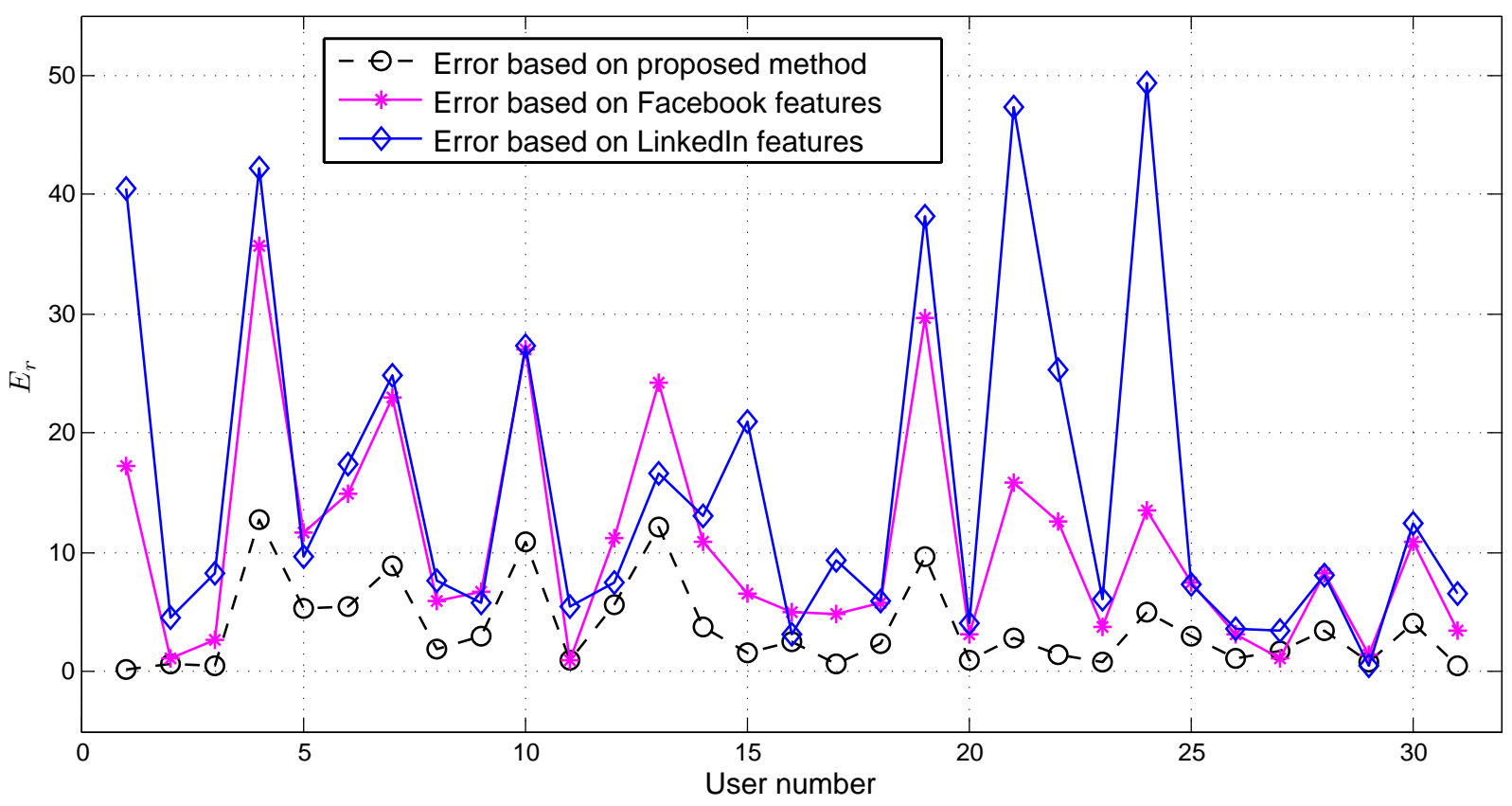

Figure 3.8: Openness Error $E_{r}$ using (i) both Facebook and LinkedIn features (Proposed method) (ii) only Facebook features and (iii) only LinkedIn features

by adding a Facebook textual feature.

In the following paragraphs, we discuss the results in detail for each personality trait. We elaborate first on the openness, conscientiousness, agreeableness and neuroticism traits and then on the extroversion trait.

Results for the openness trait: We found that the proposed method provides better results for openness rather than using only Facebook or LinkedIn. The error graph between proposed method, Facebook, and LinkedIn features is shown in Figure 3.8 ,

For Facebook, we accomplished the results as expected. In order to find the most relevant features we used a trial and testing method where all the features and their combinations were tested to get minimum error. On the other hand, after trial and testing it is noticed that the features which are moderately correlated are most relevant and provide less error for openness, such as $f_{21}$ (length of text), and $f_{23}$ (number of skills) having correlation values 0.32 , and 0.33 respectively (given in Table 
3.2. . However, we previously found a good correlation between LinkedIn features $f_{21}$ (length of text), $f_{22}$ (number of connections), $f_{23}$ (number of skills), and $f_{24}$ (number of positive words) and openness. This indicates that we can not only rely on high correlation, although it would be a preference, but in our research we test all the features and find the most relevant features.

Moreover, we have noticed a reduction in error for openness by collectively using Facebook and LinkedIn features (proposed method). The error computed is 3.71 which is much less than when using only Facebook or LinkedIn features individually, i.e 10.64 and 15.56 respectively. In the error graph we can see that in maximum cases the proposed method gives more accurate results than the other two cases except for one user (27th user) as shown in Figure 3.8. Therefore, we can say that the level of openness of a person can be computed by knowing his/her $f_{14}$ (number of likes), $f_{19}$ (number of games), $f_{21}$ (length of text) and $f_{23}$ (number of skills). Our results support some previous studies, such as [8], and [39], done using the Facebook feature number of likes. Moreover, in our study we found some new crucial features such as $f_{19}$ (number of games), $f_{21}$ (length of text), and $f_{23}$ (number of skills) which can equally contribute to the analysis of the openness trait. Furthermore, our results also support our assumptions in section 3.3.1.

Results for the conscientiousness trait We found that the Facebook feature $f_{15}$ (number of photos) gives the least error among all Facebook features as expected and postulated in previous sections. We also obtained speculated outcomes for LinkedIn. After trial and testing we observed that out of all five LinkedIn features used, only $f_{21}$ (length of text), $f_{22}$ (number of connections), and $f_{23}$ (number of skills) together provided less error than other LinkedIn features. The total error computed, by using Facebook and LinkedIn, is 12.23 \& 7.33 respectively, as given in Table 3.3 .

Furthermore, we used the proposed method and found that the combined results of Facebook and LinkedIn provide better results for conscientiousness using mode average. The error computed by combining Facebook and LinkedIn features was noted as 2.61. Therefore, it was observed that the total error $(i)$ for conscientiousness 


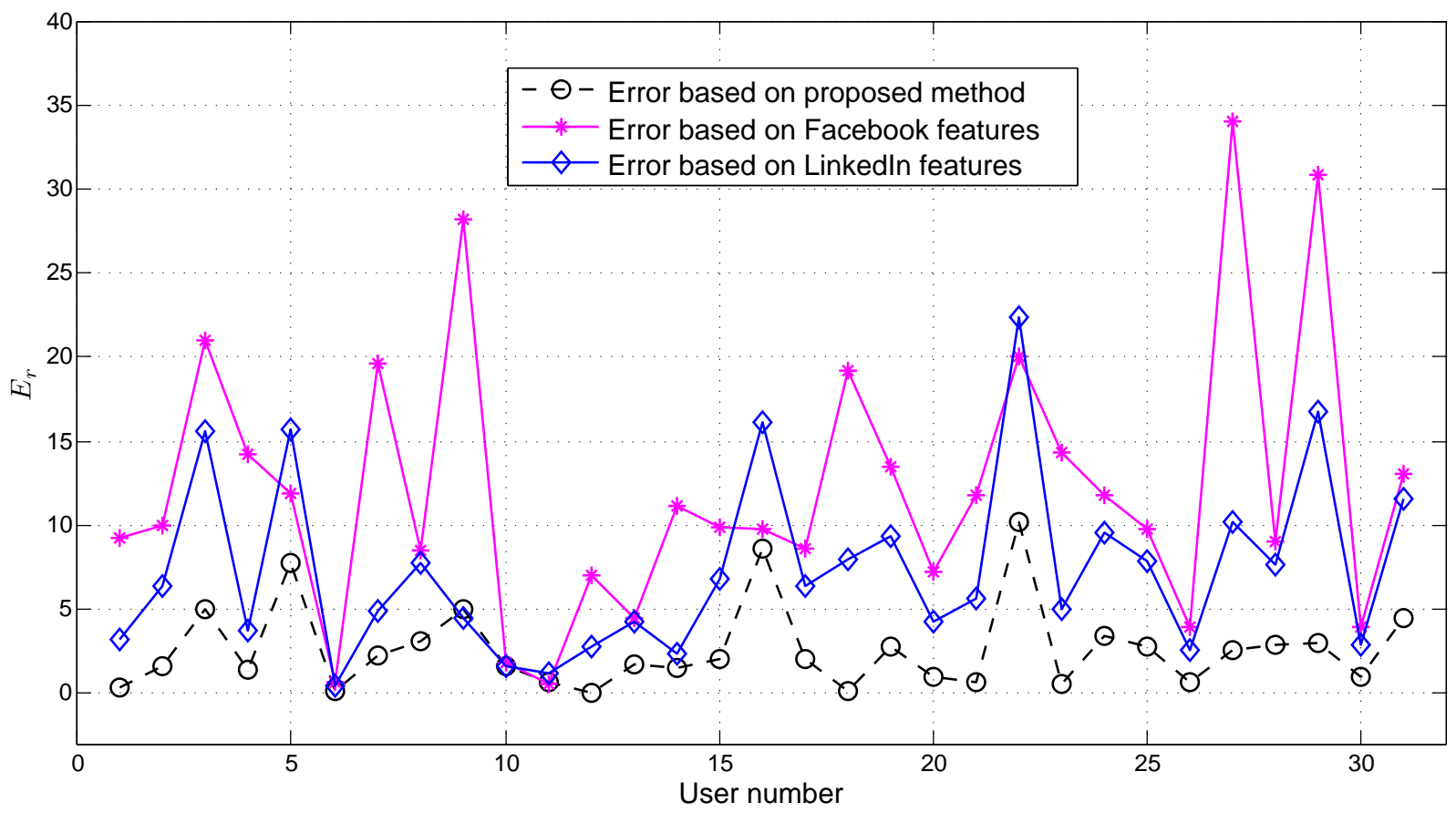

Figure 3.9: Conscientiousness Error $E_{r}$ using (i) both Facebook and LinkedIn features (ii) only Facebook features and (iii) only LinkedIn features

is reduced by $65.13 \%$ (i.e. from 10.64 to 3.71 ) by adding LinkedIn features to the feature set. Figure 3.9 shows the error $E_{r}$ graph for all 31 users using the proposed method. It can be seen in the figure that the plot showing the error using the proposed method is much closer to zero than the compared to other two options, resulting in higher accuracy.

Results for the agreeableness trait As mentioned in the previous section 3.3.2, agreeableness was found to be highly correlated with the Facebook features $f_{11}$ (number of status updates), $f_{12}$ (number of friends), and $f_{15}$ (number of photos). However, after trial and testing we noticed that only $f_{11}$ (number of status) and $f_{12}$ (number of friends) together contribute to reduce the error. Similarly, the LinkedIn feature $f_{23}$ (number of skills) provides better results than using a combination of both $f_{23}$ (number of skills) and $f_{24}$ (positive words). The error computed by incorporating these Facebook and LinkedIn feature is 3.06. We can see that this error is less than the error computed by using Facebook and LinkedIn independently i.e, $10.12 \& 15.46$ 


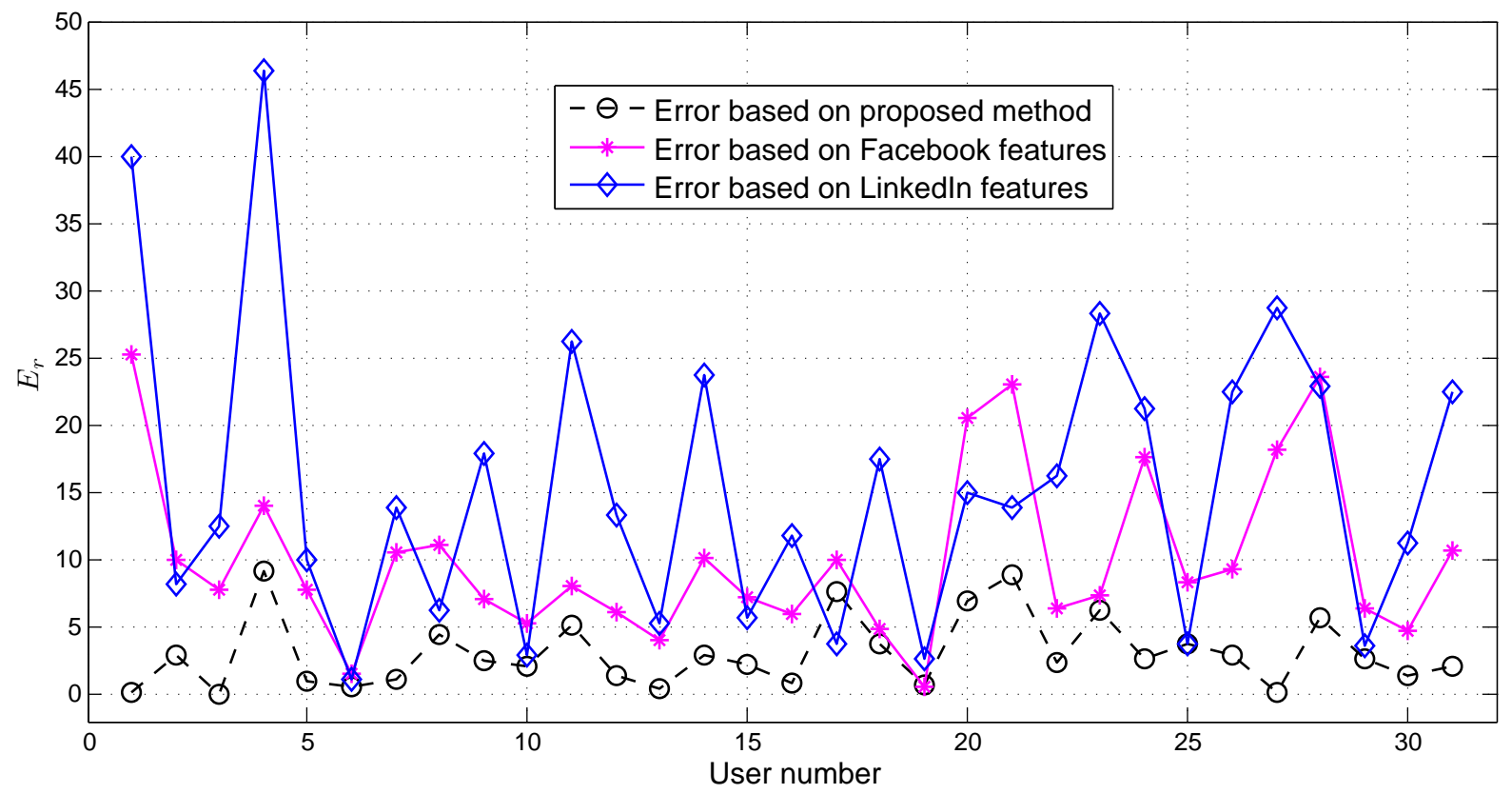

Figure 3.10: Agreeableness Error $E_{r}$ using (i) both Facebook and LinkedIn features (ii) only Facebook features and (iii) only LinkedIn features

respectively. The reduction in error for agreeableness is noted by $69.76 \%$ (i.e. from 10.12 to 3.06 ) by adding LinkedIn features to the feature set. The error graph for the proposed method, Facebook and LinkedIn is shown in Figure 3.10. We can see that the error computed by the proposed method is much closer to zero than in the other two cases. Additionally, our result is partially supported by a previous study [8] which showed that highly conscientious people have a large number of photos. On the other hand, we collected some new crucial features from LinkedIn as mentioned above.

Results for the neuroticism trait Similar to above three traits, openness, conscientiousness and agreeableness, we tested the different combinations of Facebook and LinkedIn features. We found that neuroticism was best analyzed by the Facebook features $f_{17}$ (number of events) and $f_{19}$ (number of games), and the LinkedIn features $f_{24}$ (number of positive) and $f_{25}$ (number of negative words). The error computed by integrating these Facebook and LinkedIn features (proposed) is 3.58 which is less 


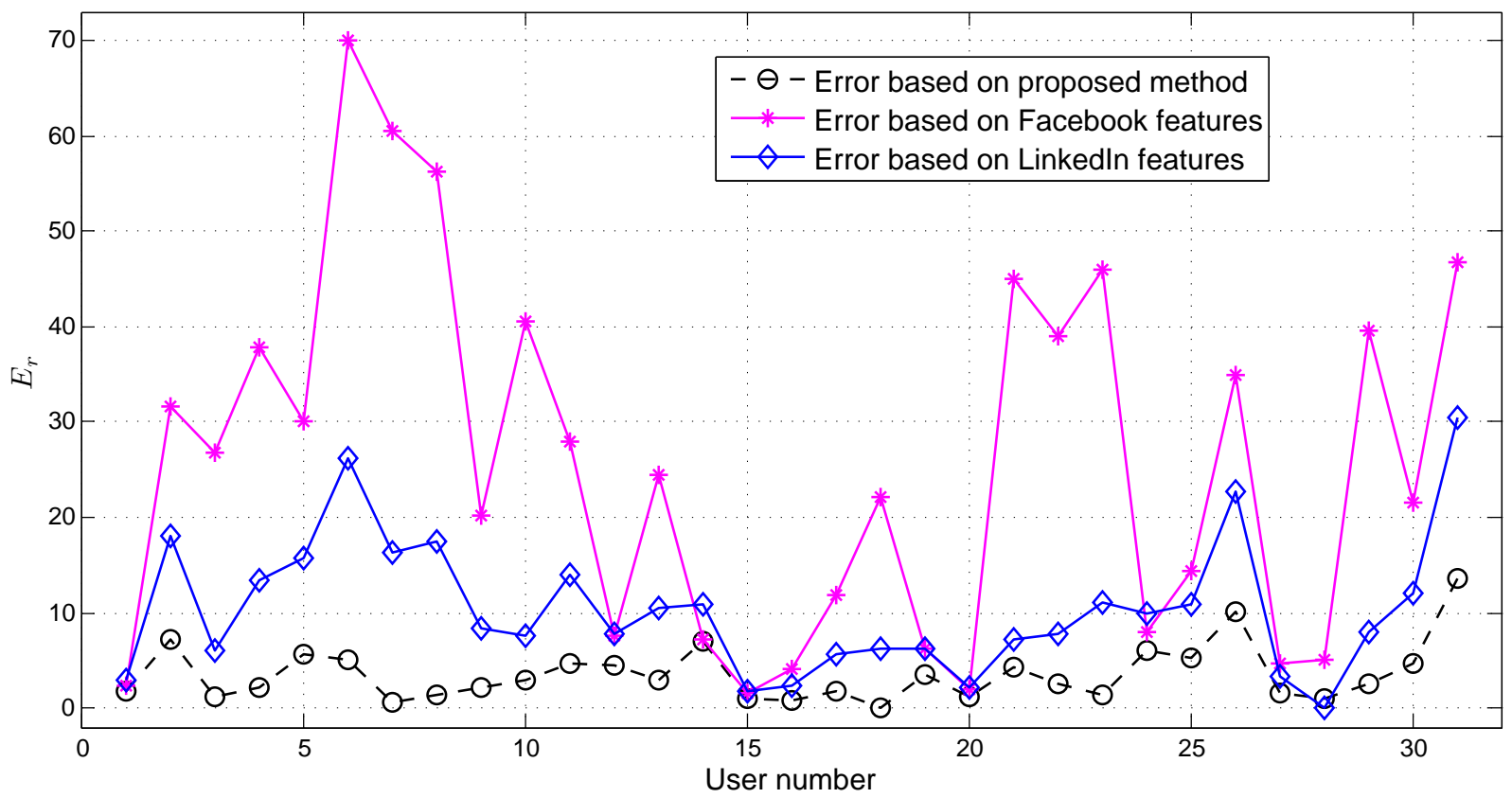

Figure 3.11: Neuroticism Error $E_{r}$ using (i) both Facebook and LinkedIn features (ii) only Facebook features and (iii) only LinkedIn features

than the other two options, using Facebook and LinkedIn, which have error of 25.69 \& 10.44. We can see the reduction in error in Table 3.3 , which is noted as $86.06 \%$ (i.e. reduced from 25.69 to 3.68) by adding LinkedIn features to the feature set. The error graph for the proposed method, Facebook, and LinkedIn is shown in Figure 3.11 where we can see that for almost each user the error is much closer to zero when using the proposed method. This confirms that combined features from multiple social network provide better results than using a single OSN, which results in higher accuracy. Our results support the outcomes of [41] which states that neurotic individuals use more negative words because they are not emotionally stable and tend be become hopeless, anxious, and hostile.

Results for the extroversion trait Unlike the above mentioned four traits, extroversion better relates to LinkedIn features than combined features of Facebook and LinkedIn, or only Facebook. We found that $f_{21}$ (length of text), $f_{22}$ (number of connections), and $f_{25}$ (number of negative words) can collectively contribute to reduce 


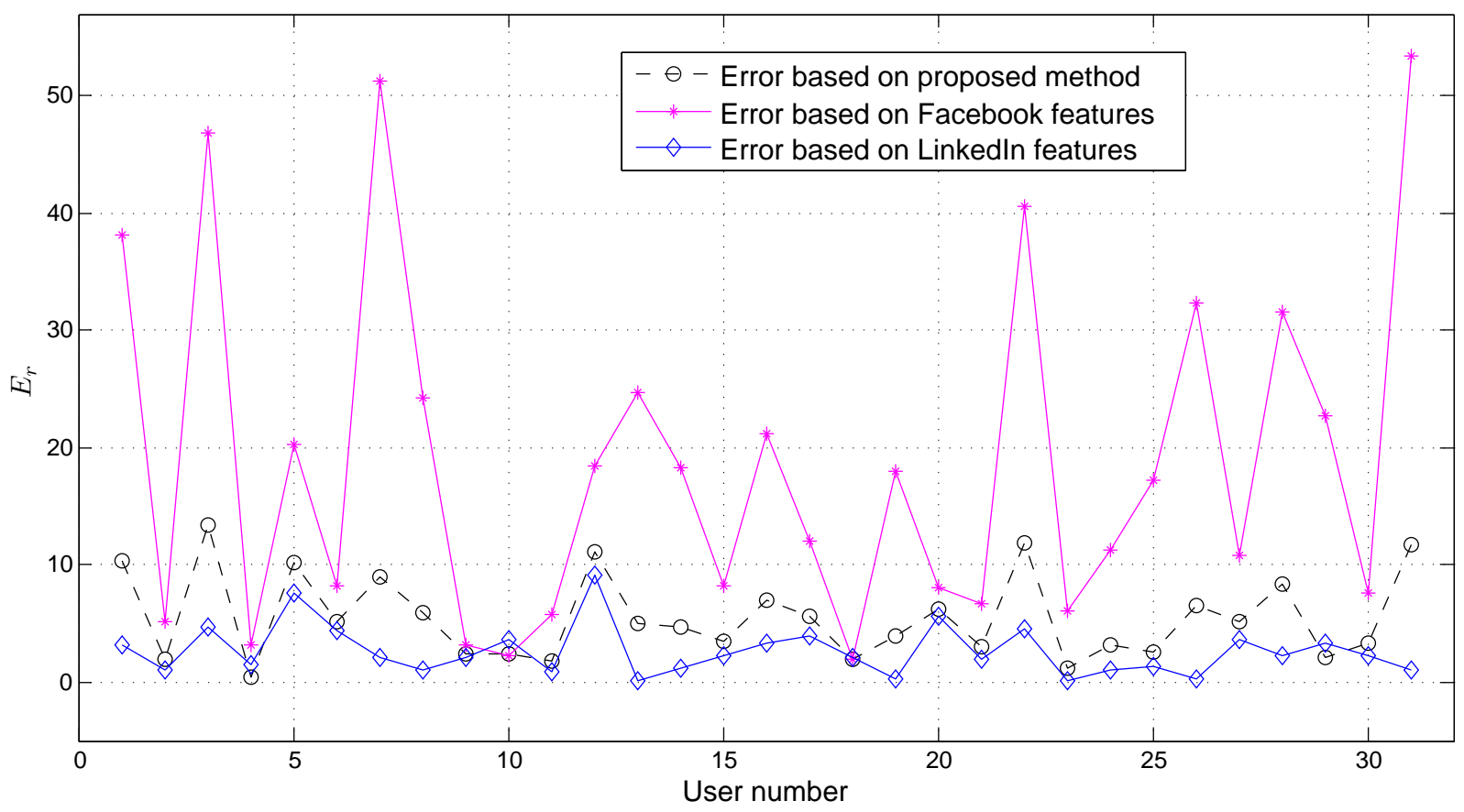

Figure 3.12: Extroversion Error $E_{r}$ using (i) both Facebook and LinkedIn features (ii) only Facebook features and (iii) only LinkedIn features

the error for extroversion. This result supports our postulates made above in section 3.3.1. While the Facebook features $f_{11}$ (number of status updates) and $f_{12}$ (number of friends) also give good results, we could not see any benefit of adding LinkedIn features to them. The total error computed by using only LinkedIn features is 2.65 which is less than the other two options: (i) proposed method, and (ii) Facebook features. The error calculated for these two options is 5.53 and 18.73 respectively. We can see the reduction in error in Table 3.3 where it is shown that the error is reduced to $69.40 \%$ (i.e, from $18.73 \%$ to $5.53 \%$ ). Our results can clearly be seen in the error graph for the proposed method, Facebook, and LinkedIn which is shown in Figure 3.12. Although we could not find any benefit of the proposed method in the case of extroversion, we were able to conclude that LinkedIn information can be equally beneficial for personality trait assessment.

Because we only considered five LinkedIn features, our research is limited to them. Therefore, there is a further need to figure out the crucial features of LinkedIn which 
Table 3.3: Error $E_{r}$ between $\hat{T}$ and $T$ using Facebook and LinkedIn, individually and collectively, for the big five personality trait trait

\begin{tabular}{|l|l|l|l|l|}
\hline Trait & $\begin{array}{l}\text { Using proposed } \\
\text { method }\end{array}$ & $\begin{array}{l}\text { Using only } \\
\text { Facebook } \\
\text { features }\end{array}$ & $\begin{array}{l}\text { Using only } \\
\text { LinkedIn fea- } \\
\text { tures }\end{array}$ & $\begin{array}{l}\text { Reduction } \\
\text { in } E_{r}\end{array}$ \\
\hline $\mathrm{O}$ & 3.71 & 10.64 & 15.56 & 65.13 \\
\hline $\mathrm{C}$ & 2.61 & 12.23 & 7.33 & 78.65 \\
\hline $\mathrm{E}$ & 5.53 & 18.72 & 2.65 & 69.40 \\
\hline $\mathrm{A}$ & 3.06 & 10.12 & 15.46 & 69.76 \\
\hline $\mathrm{N}$ & 3.58 & 25.69 & 10.44 & 86.06 \\
\hline
\end{tabular}

Table 3.4: Personality traits and relation found with features

\begin{tabular}{|l|l|l|}
\hline Trait & Facebook features & LinkedIn features \\
\hline O & Number of like, Number of games & Length of text, Number of skills \\
\hline C & Number of photos & $\begin{array}{l}\text { Length of text, Number of skills, Num- } \\
\text { ber of connections }\end{array}$ \\
\hline E & Number of status, Number of friend & $\begin{array}{l}\text { Length of text, Number of connections, } \\
\text { Number of negative words }\end{array}$ \\
\hline A & Number of status, Number of friend & Number of skills \\
\hline N & Number of events, Number of games & $\begin{array}{l}\text { Number of positive words, Number of } \\
\text { negative words }\end{array}$ \\
\hline
\end{tabular}

can minimize the error closer to zero. Table 3.4 gives a brief summary of crucial features obtained from both Facebook and LinkedIn. Also, in Table 3.3 we can see the error computed for all three options and the percentage of reduction in error for all five personality traits.

\section{Scalability}

Here we analyze the practicality of the proposed method for personality traits assessment when more than two OSNs are used. Generally speaking, it is not the number of OSNs but the total number of OSN features which adds to the computational complexity of the proposed method.

The proposed method first determines the most relevant set of features and then uses these features to compute the trait value. The former step has a computational complexity of $O\left(2^{n}\right), n$ being the number of used features. Note that it is of exponential order because 
all the features and their combinations are tested to minimize the error. The latter step is performed in linear $O(n)$ time. It is important to mention that as the former step is performed only once, it can be done in advance in an offline manner, and hence it does not have any significant impact on the practicality of the proposed method.

\subsection{Chapter Summary}

In this chapter, we have formally described the problem of personality assessment. Also, a brief description about methods involved in this research was provided which includes: i) the details about the process of information extraction from OSNs, ii) the process of feature normalization and, iii) the steps involved in modeling the personality. In addition, details about the useful experiments and results were presented. In this section, we provided details on the process of data collection obtained from the survey and OSNs with the recruitment requirements and participants. We also explained how to measure and use the OSN features. Moreover, the correlation between the five personality traits and OSN features was given for both Facebook and LinkedIn. Finally, we provided the validation for our study and results obtained from the proposed method. 


\section{Chapter 4}

\section{Conclusions and Future Research Direction}

The work presented in this thesis is aimed to identify the big five personality traits (openness, conscientiousness, extroversion, agreeableness and neuroticism) in individuals using informational OSNs. The objective of the study was to reduce the error by incorporating data gathered from multiple OSNs. We used two OSNs in our research: Facebook and LinkedIn. We found that there exists a significant relationship between the Facebook and LinkedIn features and the big five personality traits (as given in Table 3.4). However, they differ in the pattern of usage and level of effectiveness. Our study shows that the correlation between personality traits and OSN features is not straightforward as mentioned in the previous studies. We noticed that although some features have high correlation with personalities but they seem not at all beneficial to reduce the error (for example: openness was having a high negative correlation to $f_{24}$ (number of positive words), but from experiments we found that it is not beneficial to consider this feature for determining the openness trait), where the integration of some other features that had low correlation were able to reduce the error. 


\subsection{Conclusions}

This thesis makes the following conclusions:

- In addition to Facebook features, LinkedIn features such as length of text, number of connections, number of skills, and number of positive and negative words can be used to assess personality.

- The accuracy of personality assessment results (for four traits: openness, conscientiousness, agreeableness and neuroticism) improves when both Facebook and LinkedIn features are used together compared to when the features from these OSNs were used separately.

- In case of extroversion, we do not achieve any advantage of using both Facebook and LinkedIn features. Rather, extroversion trait is assessed more accurately with LinkedIn features alone compared to Facebook features only or both Facebook and LinkedIn features together.

\subsection{Future Research Direction}

Some interesting aspects for future exploration are:

1. We examined a number of Facebook and OSN features in our research, however it remains to be seen how network features such as edge centrality and k-score can impact the personality assessment results and how they can be used in our fusion model.

2. Integration of other OSNs such as Twitter and MySpace can be an interesting expansion for this work.

3. We considered the big five personality trait model for this research. Other personality trait models can be considered in the future in a multiple OSN setting. 


\section{Appendix A}

\section{Implementation Details}

Here we provide the details of implementation performed in this work. We divide the implementation in two sections: (i) Data extraction from OSNs, and (ii) Data analysis.

\section{A.1 Data Extraction}

In order to extract data we used PHP SDK and MySQL databases. Figure A.1 shows the steps involved in data extraction from Facebook and LinkedIn. First, we built a web application on Facebook and LinkedIn respectively. To create a sample application on Facebook and LinkedIn at https://developers.facebook.com/ and https://www.linkedin.com/secure/ developer respectively; we needed to take a note of the applications API Key, Secret Key, OAuth User Token, and OAuth User Secret credentials, which was then used to programmatically access the API [50]. Here, OAuth stands for 'open authorization' which provide a means to authenticate an application to allow access of account data through an API without the users needing to hand over their sensitive credentials such as username and password. There are few fundamental steps involved in the OAuth dance that ultimately enable a client application to access protected resources from the service provider on behalf of resource owner (client):

1. The client obtains an unauthorized request token from the service provider. 
2. The resource owner authorizes the request token.

3. The client exchanges the request token for an access token.

4. The client uses the access token to access protected resources on behalf of the resource owner. owner.

In this research, we use OAuth version 1.0. The description of steps involved in the data extraction are given below and can also be clearly seen in Figure A.1.

1. Create a web app on Facebook: In order to get the OSN data we first need to create a web application which can connect to Facebook and LinkedIn. In order to build an application we first need to have a valid domain (for example: www.viewmypersonality.com, etc.) which should be SSL certified. This domain will later work as a canvas for our application. In addition, a domain must have a database to save the responses. In our research we use a MySQL database in order to save the responses obtained from different users.

2. Get app id and app secret: Every application has its own Id and Secret which helps to identify the user/admin. All users has their own app Id and app secret. Therefore, they are specific for the application.

3. Get access token: There are different kinds of features available on Facebook and LinkedIn profiles. In order to get the access for each feature we sent a request to the user.

4. Get OAuth: This step involves to providing a login pop-up window. Once the user logs in he/she will be able to open the next window containing the app page. Our app provides an option to allow access to the user's data or decline. This page contains of all the information that a user is asked to share. Once the user accepts to share the data, the authorization process is complete.

5. Getting data by using API:

For Facebook: In order to get Facebook data we user Open graph API. This API 


\section{Data Extraction from OSN}

\section{Facebook}

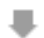

1. Create a web app on Facebook

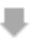

2. Get app Id and app secret

단.

3. Get access token

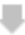

\section{Get Oauth using PHP script}

5. Use Open graph API to get data.

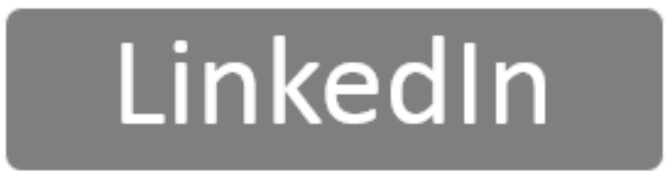

1. Create a web app on Linkedln

2. Get app Id and app secret

$v$

3. Get access token

4. Get Oauth using PHP script

단.

5. Use Rest API and get response in JSON.

Figure A.1: Steps involves in data extraction 
gives three different permissions to access a user is data as follows: (i) To get data, (ii) To post, (iii) To delete. In our research we use only to get data permission. We obtained the results of a Graph API query in a convenient 'JSON' format that can be easily manipulated and processed.

For LinkedIn: We use 'Rest API' to get LinkedIn data which can help to capture the full profile and connections of a user. We obtained the response in the 'JSON' format. Although Rest API can provide the response in the form of both 'xml' and 'JSON', but in our research we use only JSON.

The extracted data files were then converted into excel files for use in personality analysis.

\section{A.2 Data Analysis}

For data analysis we used Matlab software. It provides the tools to easily acquire, analyze and visualize the data. The data extracted from the OSNs in the form of excel files was used as a database and imported in the Matlab software. In this process, we implemented our methodology described in Section 3.2.3 in Matlab. We first calculated the Pearson

correlation between all five traits and features using the command $\operatorname{corr}(X)$. It gives a measure of the strength of the linear relation between two variables. Finally, we calculated the error between the traits obtained from the survey and the proposed method. 


\section{Appendix B}

\section{User Study}

The big five personality test questionnaire that was used in our user survey consisted of the following 50 questions:

1. Is talkative?

2. Tends to find fault with others?

3. Does a thorough job?

4. Is depressed, blue?

5. Is original, comes up with new ideas?

6. Is reserved?

7. Is helpful and unselfish with others?

8. Can be somewhat careless?

9. Is relaxed, handles stress well?

10. Is curious about many different things?

11. Is full of energy? 
12. Starts quarrels with others?

13. Is a reliable worker?

14. Can be tense?

15. Is ingenious, a deep thinker?

16. Generates a lot of enthusiasm?

17. Has a forgiving nature?

18. Tends to be disorganized?

19. Worries a lot?

20. Has an active imagination?

21. Tends to be quiet?

22. Is generally trusting?

23. Tends to be lazy?

24. Is emotionally stable, not easily upset?

25. Is inventive?

26. Has an assertive personality?

27. Can be cold and aloof?

28. Perseveres until the task is finished?

29. Can be moody?

30. Values artistic, aesthetic experiences?

31. Is sometimes shy, inhibited?

32. Is considerate and kind to almost everyone? 
33. Does things efficiently?

34. Remains calm in tense situations?

35. Prefers work that is routine?

36. Is outgoing, sociable?

37. Is sometimes rude to others?

38. Makes plans and follows through with them?

39. Gets nervous easily?

40. Likes to reflect, play with ideas?

41. Has few artistic interests?

42. Likes to cooperate with others?

43. Is easily distracted?

44. Is sophisticated in art, music, or literature?

45. Is politically liberal?

46. Your gender?

47. How old are you?

48. Number of years of formal education you have completed?

49. How much do you enjoy your profession?

50. How long have you been involved with your profession?

Note that questions 1 to 47 were mandatory to answer whereas questions 48 to 50 were optional. This online test uses Likert scale to rate the answers which is given as: Strongly agree, Agree, Not sure, Disagree, Strongly disagree. This psychological big five personality test is available online freely at http://www.outofservice.com/bigfive/ 


\section{References}

[1] Big five personality factor. http://http-server.carleton.ca/ tpychyl/ 011382000/BigFive.html.

[2] Yair Amichai-Hamburger. Internet and personality. Computers in Human Behavior, 18(1):1-10, 2002.

[3] Yair Amichai-Hamburger. Personality and the internet. The social net: Human behavior in cyberspace, pages 27-55, 2005.

[4] Yair Amichai-Hamburger and E. Ben-Artzi. The relationship between extraversion and neuroticism and the different uses of the internet. Computers in Human Behavior, 16(4):441-449, 2000.

[5] Yair Amichai-Hamburger, Hadar Kaplan, and Nira Dorpatcheon. Click to the past: The impact of extroversion by users of nostalgic websites on the use of internet social services. Computers in Human Behavior, 24(5):1907-1912, 2008.

[6] Yair Amichai-Hamburger and Gideon Vinitzky. Social network use and personality. Computers in Human Behavior, 26(6):1289-1295, 2010.

[7] Association for Psychological Science. Marketing is more effective when targeted to personality profiles. ScienceDaily. 21 May 2012. http://www.sciencedaily.com/ releases/2012/05/120521115652.htm.

[8] Yoram Bachrach, Michal Kosinski, Thore Graepel, Pushmeet Kohli, and David Stillwell. Personality and patterns of facebook usage. In Proceedings of the 3rd Annual ACM Web Science Conference, pages 24-32, Evanston, IL, USA, 2012. 
[9] Mitja D Back, Juliane M Stopfer, Simine Vazire, Sam Gaddis, Stefan C Schmukle, Boris Egloff, and Samuel D Gosling. Facebook profiles reflect actual personality, not self-idealization. Psychological Science, 21(3):372-374, 2010.

[10] Shuotian Bai, Tingshao Zhu, and Li Cheng. Big-five personality prediction based on user behaviors at social network sites. arXiv preprint arXiv:1204.4809, 2012.

[11] Zuzana Birknerová, Radovan Bačík, and Jaroslava Gburová. The effectiveness of advertising in relation to the personality of the consumer. Journal of Finance and Economics, 1(2):17-21, 2013.

[12] Jack Block. A contrarian view of the five-factor approach to personality description. Psychological Bulletin, 117(2):187, 1995.

[13] Tim Blumer and Nicola Döring. Are we the same online? The expression of the five factor personality traits on the computer and the Internet. Cyberpsychology: Journal of Psychosocial Research on Cyberspace, 6(3), 2012.

[14] Nathan Brody and Howard Ehrlichman. Personality psychology: The science of individuality, volume 3. Prentice Hall Englewood Cliffs, NJ, 1998.

[15] Vittorio V Busato, Frans J Prins, Jan J Elshout, and Christiaan Hamaker. Intellectual ability, learning style, personality, achievement motivation and academic success of psychology students in higher education. Personality and Individual Differences, 29(6):1057-1068, 2000.

[16] Gian Vittorio Capara, Claudio Barbaranelli, and Philip G. Zimbardo. Personality profiles and political parties. Political Psychology, 20(1):175-197, 1999.

[17] Tomas Chamorro-Premuzic. Personality and individual differences. Blackwell publishing, 2007.

[18] Kendra Cherry. Trait theory of personality. http://psychology.about.com/od/ theoriesofpersonality/a/trait-theory.htm. Accessed on June 28, 2014. 
[19] Teresa Correa, Amber Willard Hinsley, and Homero Gil De Zuniga. Who interacts on the web?: The intersection of users personality and social media use. Computers in Human Behavior, 26(2):247-253, 2010.

[20] Paul T Costa and Robert R McCrae. Personality in adulthood: a six-year longitudinal study of self-reports and spouse ratings on the neo personality inventory. Journal of Personality and Social Psychology, 54(5):853, 1988.

[21] Paul T Costa and Robert R McCrae. Normal personality assessment in clinical practice: The neo personality inventory. Psychological Assessment, 4(1):5, 1992.

[22] Randy Duermyer. Introduction to LinkedIn, How LinkedIn works. http:// homebusiness.about.com/od/linkedin/a/how-does-linkedin-work.htm, 2014.

[23] Duggan and Smith. Social media update . http://www.pewinternet.org/2013/12/ 30/social-media-update-2013/, Dec 2013.

[24] Hans J Eysenck. Dimensions of personality. Springer, 1991.

[25] Global Faces and Networked Places. A Nielsen report on social networkings new global footprint, March 2009. http://blog.nielsen.com/nielsenwire/wp-content/ uploads/2009/03/nielsen_globalfaces_mar09.pdf.

[26] Global Faces and Networked Places. What do personality traits tell us about consumer behavior? http://www.beyondthepurchase.org/blog/01/ what-do-personality-traits-tell-us-about-consumer-behavior/, 2014.

[27] Don C Fowles. Application of a behavioral theory of motivation to the concepts of anxiety and impulsivity. Journal of Research in Personality, 21(4):417-435, 1987.

[28] Jennifer Golbeck, Cristina Robles, Michon Edmondson, and Karen Turner. Predicting personality from twitter. In Proceedings of the Third IEEE International Conference on Privacy, Security, Risk and Trust (PASSAT) and Third IEEE International Conference on Social Computing (SocialCom), pages 149-156, Boston, MA, USA, 2011. 
[29] Jennifer Golbeck, Cristina Robles, and Karen Turner. Predicting personality with social media. In Proceedings of the ACM CHI'11 Extended Abstracts on Human Factors in Computing Systems, pages 253-262, Vancouver, BC, Canada, 2011.

[30] Rosanna E Guadagno, Bradley M Okdie, and Cassie A Eno. Who blogs? Personality predictors of blogging. Computers in Human Behavior, 24(5):1993-2004, 2008.

[31] Elizabeth Hardie and Ming Yi Tee. Excessive internet use: The role of personality, loneliness and social support networks in internet addiction. Australian Journal of Emerging Technologies and Society, 5(1), 2007.

[32] Curt Haugtvedt, Richard E Petty, John T Cacioppo, and Theresa Steidley. Personality and ad effectiveness: Exploring the utility of need for cognition. Advances in Consumer Research, 15, 1988.

[33] Jacob B Hirsh, Colin G DeYoung, Xiaowen Xu, and Jordan B Peterson. Compassionate liberals and polite conservatives: Associations of agreeableness with political ideology and moral values. Personality and Social Psychology Bulletin, 36(5):655-664, 2010.

[34] Jacob B Hirsh, Sonia K Kang, and Galen V Bodenhausen. Personalized persuasion tailoring persuasive appeals to recipients personality traits. Psychological Science, 23(6):578-581, 2012.

[35] Martin Holzwarth, Chris Janiszewski, and Marcus M Neumann. The influence of avatars on online consumer shopping behavior. Journal of Marketing, 70(4):19-36, 2006.

[36] Francisco Iacobelli, Alastair J Gill, Scott Nowson, and Jon Oberlander. Large scale personality classification of bloggers. In Proceedings of the Affective Computing and Intelligent Interaction, pages 568-577. Springer, 2011.

[37] Oliver D. John. Big five personality test. http://www.outofservice.com/bigfive/, 2000. 
[38] Timothy A Judge and Joyce E Bono. Five-factor model of personality and transformational leadership. Journal of Applied Psychology, 85(5):751-765, 2000.

[39] Milutinovi V Kartelj A., Filipovi V. Novel approaches to automated personality classification: Ideas and their potentials. In Proceedings of the 35th International Convention, MIPRO, pages 1017-1022. IEEE, 2012.

[40] Sanjay Kumar. The concept of personality. Recent Receptive Exploration Journal, $1(3): 54-56$.

[41] Randy J Larsen and Timothy Ketelaar. Extraversion, neuroticism and susceptibility to positive and negative mood induction procedures. Personality and Individual Differences, 10(12):1221-1228, 1989.

[42] Randy J Larsen and Timothy Ketelaar. Personality and susceptibility to positive and negative emotional states. Journal of Personality and Social Psychology, 61(1):132, 1991.

[43] Lei Li, Yang Yang, and Liu Mingxin. The relationship between adolescents' neuroticism, internet service preference, and internet addiction. Acta Psychologica Sinica, 3:008, 2006.

[44] Mashable. "facebook". http://mashable.com/category/facebook/.

[45] Mehwash Mehroof and Mark D Griffiths. Online gaming addiction: The role of sensation seeking, self-control, neuroticism, aggression, state anxiety, and trait anxiety. Cyberpsychology, Behavior, and Social Networking, 13(3):313-316, 2010.

[46] James W Pennebaker, Matthias R Mehl, and Kate G Niederhoffer. Psychological aspects of natural language use: Our words, our selves. Annual Review of Psychology, 54(1):547-577, 2003.

[47] Daniele Quercia, Michal Kosinski, David Stillwell, and Jon Crowcroft. Our twitter profiles, our selves: Predicting personality with twitter. In Proceedings of the Third IEEE International Conference on Privacy, Security, Risk and Trust (PASSAT) and 
Third IEEE International Conference on Social Computing (SocialCom), pages 180185, Boston, MA, USA, 2011.

[48] Brent W Roberts, Nathan R Kuncel, Rebecca Shiner, Avshalom Caspi, and Lewis R Goldberg. The power of personality: The comparative validity of personality traits, socioeconomic status, and cognitive ability for predicting important life outcomes. Perspectives on Psychological Science, 2(4):313-345, 2007.

[49] Craig Ross, Emily S Orr, Mia Sisic, Jaime M Arseneault, Mary G Simmering, and R Robert Orr. Personality and motivations associated with facebook use. Computers in Human Behavior, 25(2):578-586, 2009.

[50] Matthew A Russell. Mining the Social Web: Data Mining Facebook, Twitter, LinkedIn, Google+, GitHub, and More. " O’Reilly Media, Inc.", 2013.

[51] Stephanie V Stone and Paul T Costa Jr. Disease-prone personality or distress-prone personality? The role of neuroticism in coronary heart disease. In Howard S. Friedman, editor, Proceedings of the Personality and Disease, Oxford, 1990. John Wiley \& Sons.

[52] Kil-Soo Suh, Hongki Kim, and Eung Kyo Suh. What if your avatar looks like you? Dual-congruity perspectives for avatar use. MIs Quarterly, 35(3):711-729, 2011.

[53] Yla R Tausczik and James W Pennebaker. The psychological meaning of words: Liwc and computerized text analysis methods. Journal of Language and Social Psychology, 29(1):24-54, 2010.

[54] Tracy L Tuten and Michael Bosnjak. Understanding differences in web usage: The role of need for cognition and the five factor model of personality. Social Behavior and Personality: An International Journal, 29(4):391-398, 2001.

[55] A. Verschuren. Relying on linkedin profiles for personality impressions. Master's thesis, Tilburg University, The Netherlands, 2012.

[56] Randall Wald, Taghi Khoshgoftaar, and Chris Sumner. Machine prediction of personality from facebook profiles. In Proceedings of the 13th International Conference on Information Reuse and Integration (IRI), pages 109-115. IEEE, 2012. 
[57] Tal Yarkoni. Personality in 100,000 words: A large-scale analysis of personality and word use among bloggers. Journal of Research in Personality, 44(3):363-373, 2010. 